\title{
Dinámica fluvial durante el inicio del Holoceno superior en el curso medio del Vinalopó (Alicante, España)
}

\author{
C. Ferrer García \\ Museu de Prehistòria de València, Centro Cultural La Beneficència, Carrer de la Corona 36, E-46003 València, España \\ carlos.ferrer@dival.es
}

\begin{abstract}
RESUMEN
El estudio geomorfológico y estratigráfico de una serie de perfiles del sistema de terrazas fluviales del curso medio del Vinalopó y el análisis sedimentológico textural de los depósitos que conforman el yacimiento arqueológico de EDAR de Monforte del Cid (siglo I a. C.), permiten conocer la dinámica sedimentaria del medio durante la primera parte del Holoceno superior (del 5000 a. C. al 400 d. C.). A su vez, la puesta en relación de esta dinámica con los datos derivados del estudio arqueológico acerca de la ocupación del territorio por parte de las sociedades antiguas, hace posible establecer las posibles influencias antrópicas sobre el medio físico. El sistema fluvial, en un contexto climático semiárido del sureste peninsular, posee una compleja evolución, en la que destacan los procesos de agradación sedimentaria, en particular entre los siglos VIV a. C. y III-IV d. C. Intercalada se documenta una extensa fase, en un contexto climático algo más húmedo que el actual, en la que alternan periodos en los que procesos deposicionales se ralentizan en mayor o menor medida. Este comportamiento desigual, al menos a partir del periodo ibero-romano, siglo III a. C., parece tener una relación directa con la presión humana sobre la cuenca. Esta aproximación geoarqueológica aporta datos relevantes a la discusión en torno al impacto de las actividades humanas en la dinámica de los medios sedimentarios fluviales.
\end{abstract}

Palabras clave: Estratigrafía, Geoarqueologia, Holoceno superior, sedimentación fluvial, Sedimentología.

\section{Fluvial dynamics during the Early Upper Holocene in the Vinalopó middle watercourse (Alicante, Spain)}

\begin{abstract}
This paper presents the geomorphological and stratigraphical study of several sequences of fluvial terraces in the centre of the Vinalopó River and the sedimentological analysis (grain size) of depositional sequences in the archaeological site of EDAR in Monforte del Cid ( $1^{\text {st }}$ century BC). Both studies allow us to pinpoint fluvial sedimentary dynamics during the first part of the upper Holocene (5000 BC-400 AD) in an arid environment in south-eastern Spain. The analysis of the territory occupation allows us to relate sedimentary processes with human activity. The fluvial system has had a complex sedimentary evolution. Sedimentary aggradation processes are relevant, particularly from the $5^{\text {th }}-4^{\text {th }}$ century $B C$ up to the $3^{\text {rd }}-4^{\text {th }}$ century $A D$. Between these periods slow depositional processes alternated with quasi-stability phases are documented, in a more humid climate than the current context. These geomorphological processes appear to have had a direct relationship with the level of human pressure on the basin, at least since the third century BC. This modest geoarchaeological approach provides relevant data to the discussion about the impact of human activitiy on the fluvial sedimentary dynamics.
\end{abstract}

Key words: fluvial sedimentation, Geoarchaeology, river terraces, sedimentology, stratigraphy, Upper Holocene.

ABRIDGED ENGLISH VERSION

Introduction

Fluvial deposits are records of sedimentary processes and environmental conditions of the past. Their geo- 
Ferrer García, C., 2018. Dinámica fluvial durante el inicio del Holoceno superior en el... Boletín Geológico y Minero, 129 (1/2): $305-330$

morphological, chronostratigraphic and sedimentological study, amongst other methods, is useful in establishing paleo-environmental and geoarchaeological sequences, either documented episodes of climatically significant fluvial activity, sometimes associated with rapid climate change, or the analysis of the influence of human activity on these systems.

The aim of this study is to obtain an understanding of the dynamics in the fluvial sedimentary basin of the river Vinalopó in the Holocene period which extended from at least 5,0 ky cal BP up to the third century BC. The geoarchaeological study of a small area of its central course provides data which, together with knowledge of the historical settlement, allows us to answer the question of how far the river dynamics in this environment have been conditioned by human impact in recent millennia.

This paper deals with the study of the stratigraphy of later river terraces in the central Vinalopó valley and the sedimentological analysis of the stratigraphic units at the archaeological site EDAR in Monforte del Cid, which is also integrated in the fluvial sequence. This study has allowed us to identify the sedimentary processes and the evolution of the landscape during a long period of the upper Holocene up to the third century BC (Segura et al., 2012).

\section{Study Area}

The river Vinalopó is a seasonal fluvial course of low and irregular water levels with a braided riverbed. The drainage area is located in south-eastern Iberia, in the western Mediterranean region. This is a semi-arid climate zone.

The Vinalopó valley is formed by a series of basins separated by limestone ranges of prebetic orientation, WSWIENE. The river flows through the tectonic accident of the Vinalopó fault and associated Triassic clays and goes across the above mentioned reliefs (Rodríguez-Estrella, 1977, Alfaro et al., 2004). The study area is located in the floodplain of the Agost basin that forms the river in the middle of the Vinalopó, before reaching the anticline of Serra Crevillent, in the confluence of two tributaries.

This area has had an intense human occupation throughout recent Prehistory. Human pressure is intensified in the Metal Age. During the Campaniforme, around 5,000 years ago, and the Bronze Age, 4,000 years ago, more than 22 settlements have been catalogued. These sites show exploitation of the plains with agricultural activities (Hernández Pérez, 1983, Jover Maestre and López Padilla, 2004, Molina Mas and Ortega Pérez, 2010).

The occupation was also significant between the fourth to sixth centuries BC (Abad Casal and Alberola Belda, 1990, Abad Casal and Sala Selles, 1992, Abad Casal et al., 1998, Mas and Ortega Perez Molina, 2010). A depopulation of the area between the third and first centuries BC has been proposed (Tendero Porras 2014, Molina Vidal, 2002). At the turn of era they have proposed major changes in the structure of the settlement. The reorganization of the territory according to the Roman model would have occurred in the immediately following century (Moratalla, 2001). Finally, in the late Imperial Roman Period the occupation of this territory appears to have been very intense, particularly from the fourth century BC (Tendero Porras et al., 2014).

\section{Methods}

The sedimentary records have been geomorphologically, stratigraphically and sedimentologically studied. Their evolution over time is based on their relative dating from the contextual information provided by the archaeological record. The correlation of the paleo-environmental data with archaeological data has allowed us to address the problem of the interaction between the environment and human communities.

\section{Results}

Fluvial terraces

A system of three successive terraces has been documented in the study area. The upper two terraces are respectively dated to the late Upper Pleistocene and to the Late Glacial. At the top of the second one, which constitutes most of the floodplain, sand and edaphic formations overlap betraying a contrasted environmental dynamic during the first half of the Holocene. Terrace T3, dated to the Upper Holocene period, is located at around $6 / 5 \mathrm{~m}$ above the talweg of the Vinalopó river and it forms the lower part of the floodplain. It is made up of four units of braided sedimentary features. The first is dated after the Bronze Age, from 4,000 BP onwards. The second is dated to the lberian period, 2,500 BP onwards. The third unit is later than the late Roman period, from 1,200 BP onwards. In the base of this unit there is a potent and continuous level of debris 
Ferrer García, C., 2018. Dinámica fluvial durante el inicio del Holoceno superior en el... Boletín Geológico y Minero, 129 (1/2): $305-330$

flow. The fourth unit is dated before the medieval period. Broadly speaking, the suggested sequence seems to coincide with current palaeo-environmental models, where aggradation processes have been identified from 5,000 BP and which intensify before 3,000 BP (Martínez Cortizas et al., 2005, Fletcher and Sanchez Goñi, 2008, amongst others). This model is also coherent with what has been documented in other fluvial systems in semi-arid environments (Uribelarrea and Benito, 2008, Calmel-Avila et al., 2009, Constante et al., 2011). However, this dynamic is not homogeneous, as intermediate phases of stability also seem to have been identified.

The archaeological site of EDAR

The site is located on an interfluve next to the river channels of the Vinalopó valley and the "rambla" of EI Orito. The remains of a building dating to 100/80 BC constitute the main archaeological feature recovered during the excavations. The foundations of the building are laid out on fluvial sands (level I) and on grey silt and clay sediments that show hydromorphic soil processes (level II). The latter layer covers level I and is dated to the $2^{\text {nd }}$ century $B C$.

Following the archaeological excavation of the site, an interpretation in terms of a ritual use of this building has been suggested in relation to the groundwater upwelling that would explain the hydromorphic features recorded in level II. Between 90 and 40 BC the clearing out of the area has been identified, as well as its colmatation with sediments similar to those identified in the ritual context (level III). After the destruction of the site at around 50-40 BC a series of decimetric layers of fluvial sands associated to slow accretion processes were deposited (levels IVa and IVb, dated during the second half of the $1^{\text {st }}$ century $A D$ and to between the $1^{\text {st }}$ and $2^{\text {nd }}$ centuries $\left.A D\right)$. They were covered by grey silt and clay sediments of the floodplain that eventually sealed the site by the $3^{\text {rd }}$ century $A D$ (level $V$ ).

\section{Discussion: Recent fluvial dynamics}

The period stretching from 4,000 to 1,500 BP is a phase of active fluvial morphogenesis in the middle Vinalopó valley. This feature is related to unfavourable environmental conditions due to climatic reasons and with the A morphogenetic phase of low activity on the main riverbed has been documented with certainty, at least from the $3^{\text {rd }}$ century $B C$ and up to the $2^{\text {nd }}$ century $A D$. In the archaeological site this phase is detected in the formation of sand bars and riverbeds associated to slow aggradation processes and in the formation of silt and clay deposits, the result of decantation processes in hydromorphic environments on the lower terraces around the $2^{\text {nd }}$ and $1^{\text {st }}$ centuries $B C$. Similar sedimentary processes have occurred in other Mediterranean areas during favourable climatic conditions, in the context of low human activity and in relation to changes to landscape exploitation (Calmel-Avila, 2000, Faust et al., 2000, 2004 y 2015, Carrión et al., 2003, Schulte, 2002, Benito et al., 2008, Sancho et al., 2008, Schulte et al., 2008, Uribellarea and Benito, 2008, Zielhofer and Faust, 2008, García-Ruiz, 2010, Constante et al., 2010, Currás et al., 2012, Wolf et al., 2013). According to the archaeological data, there would have been lower human pressure during this period (Moratalla, 2005), which would explain the low rates of aggradation in connection to the recovery of the soil in the basin following the reduction of agricultural land.

Afterwards, the reactivation of aggradation fluvial sedimentary processes would have constructed the medium segment of terrace T3. On the riverbed, a debris flow deposit documented on the structures is dated to the $2^{\text {nd }}$ century $A D$, and sealed with a layer with pottery from the $3^{\text {rd }}$ century $A D$, and is likely to be related to a very active morphogenetic phase dated to the late Roman Imperial period, although there is admittedly little evidence of this feature in south-eastern Iberia so far. All in all, this seems to coincide with a moment of higher human pressure on the landscape dating from the $4^{\text {th }}$ century AD (Tendero et al., 2014).

\section{Introducción}

Los depósitos fluviales constituyen registros de los procesos sedimentarios y de las condiciones ambientales del pasado. Su estudio geomorfológico, cronoestratigráfico y sedimentológico, entre otros métodos, es particularmente útil en el establecimiento de secuencias paleoambientales y geoarqueológicas
(Stokes et al., 2012, Cordier et al., 2014), ya sea documentando episodios de actividad fluvial climáticamente significativos, en ocasiones asociados a cambios climáticos rápidos, ya sea en el análisis de la influencia de la actividad humana en estos sistemas (Benito et al., 2006, 2008 y 2013, Uribelarrea and Benito, 2008, Gregory et al., 2006, Maklin et al., 2005 y 2012, Thorndycraft y Benito, 2006 y Faust et al., 2015). 
Ferrer García, C., 2018. Dinámica fluvial durante el inicio del Holoceno superior en el... Boletín Geológico y Minero, 129 (1/2): $305-330$

La antropogénesis fue considerada clave en el origen de paisajes tan humanizados como los mediterráneos a finales del siglo XX (Butzer, 2005). La actividad humana sería perceptible en el medio físico a partir de la introducción de las actividades productivas en los territorios, que habría dado lugar a cambios en la cubierta vegetal, en los sistemas hidrológicos y edáficos (Faust et al., 2004, Ramos Muñoz and Pérez Rodríguez, 2008, Roberts et al., 2011). Destacan los trabajos pioneros en torno a medios fluviales de Vita Finci (1969) y Van Zuidam (1975), continuados por Butzer (Butzer 1982) y Van Andel (Van Andel et al., 1986; Van Andel et al., 1990), así como los llevados a cabo en laderas (Burillo and Peña, 1984 y Burillo et al., 1985). En los últimos años, a partir de la constatación del elevado impacto a escala humana de los cambios climáticos, los paradigmas han variado significativamente: han puesto al sistema terrestre muy por encima de la capacidad de cambio del ser humano (Cordier et al., 2014).

En el Mediterráneo occidental empieza a documentarse un impacto poco relevante de la acción humana sobre la vegetación en torno al 7,0 ka cal BP (Dupré, 1995, Pérez-Obiol et al., 2011, Badal et al., 2012, Zapata et al., 2013, Marinova et al., 2012, Revelles et al., 2015). Tras un periodo para el que es difícil discernir la actividad humana o el clima como factores primarios (Thordycarf and Benito, 2006, Zielhofer et al., 2008, Roberts et al., 2011), la presión humana habría dado lugar a la degradación forestal en las cuencas bajas del mediterráneo peninsular de forma evidente a partir del $5 \mathrm{ka}$ cal BP (Jalut et al., 1997 y 2000, Carrión et al., 2007). Su influencia habría sido significativa desde el 4,5 ka cal BP (Julià et al., 2000, Jalut et al., 2009, Bal et al., 2011, Rubiales et al., 2012, Ferme et al., 2014), e intensa en época argárica, al menos en el Sureste (Carrión et al., 2010), en relación con el incremento de los fuegos forestales que se habría producido aproximadamente entre el 4,0 y 3,0 ka cal BP (García-Alix et al., 2013). Ahora bien, todavía está en cuestión cuándo el impacto de las actividades humanas afecta de forma significativa a la totalidad de las cuencas e influye en la dinámica de los sistemas geomorfológicos fluviales y aluviales, y en qué sentido lo hace (Gutiérrez-Elorza and PeñaMonné, 1998, Faust et al., 2000 y 2004, Zielhofer and Faust, 2008, García-Ruiz, 2010, Carmona and Ruiz, 2011, Constante et al., 2010 y 2011, y Wolf et al., 2014).

Los periodos Ibérico y Romano parecen ser claves en los cambios producidos en el medio como resultado de la acción humana (Ejarque et al., 2010, MartínPuertas et al., 2010, Fletcher et al., 2013). Algunos estudios proponen que durante fases de rasgos climáticos húmedos se darían condiciones de elevada o baja intensidad en la dinámica sedimentaria en función de un mayor o menor impacto humano (Bellin et al., 2013); otros en cambio defienden que esta influencia habría consistido básicamente en la amplificación de la degradación de la cubierta vegetal y de los procesos geomorfológicos durante periodos áridos (Wolf et al., 2013).

El objetivo de este trabajo es obtener una mejor comprensión de la dinámica sedimentaria fluvial en la cuenca del rio Vinalopó en un periodo del Holoceno superior que se extiende desde al menos el 5,0 ka cal BP hasta el siglo IV d. C. El estudio geoarqueológico de una pequeña área de su curso medio aporta datos que, unidos al conocimiento del poblamiento histórico, permiten responder a la cuestión de en qué medida la dinámica fluvial en este ambiente han estado condicionada por el impacto humano en los últimos milenios, y en qué sentido se han producido.

\section{Zona de estudio}

\section{Marco geográfico}

El espacio estudiado ocupa un área de un $1 \mathrm{~km}^{2}$ en la partida de las Agualejas del Ilano de Monforte del Cid, en la cuenca media del llamado valle del Vinalopó, en el interior de las comarcas meridionales valencianas. Incluye una serie de niveles de terraza fluvial, uno de los cuales integra en su secuencia sedimentaria el yacimiento arqueológico de la estación depuradora de aguas residuales (EDAR).

Desde el punto de vista climático la cuenca del Vinalopó se emplaza en un ámbito mediterráneo de características semiáridas, a sotavento de las sierras béticas valencianas; con precipitaciones por debajo de los $350 \mathrm{~mm}$ de media anual (Clavero, 1977, Pérez Cueva, 1994). Rasgo que se va pronunciado conforme nos desplazamos al sur. Es el caso de Monforte, donde estas se sitúan en torno a los $282 \mathrm{~mm}$ de media anual (Gil Olcina, 2009). Esta semiaridez da lugar a exiguos caudales: un módulo de $0,3 \mathrm{~m}^{3} / \mathrm{s}$ a la altura de Monforte, a pesar de que el río posee una extensa cuenca de más de $1.670 \mathrm{~km}^{2}$ (Rosselló and Verger and Boira and Maiques, 1995). Ello no es óbice para que históricamente se hayan producido lluvias de fuerte intensidad horaria que dieron lugar a inundaciones con ondas de crecida que superan los límites del cauce encajado, en particular en aquellos eventos que superan los $100 \mathrm{~mm} / \mathrm{m}^{2}$ (Olcina Cantos et al., 2007).

El valle del Vinalopó está formado por una sucesión de depresiones y cuencas separadas por relieves calcáreos de orientación prebética (SE-NO). El accidente o falla de desgarre del Vinalopó (Rodríguez- 
Estrella, 1977), al que se asocia una alineación diapírica triásica de dirección transversal a las depresiones, NO-SE, es el elemento tectónico que le caracteriza y da forma. Funciona como línea de debilidad que el río Vinalopó aprovecha para comunicar las sucesivas fosas atravesando los horts y anticlinales que las separan. Esta especial configuración, unida a la intensa actividad neotectónica diapírica (Alfaro et al., 2004) y a los rasgos climáticos, dan lugar a espacios endorreicos en zonas marginales, que fueron más extensas en algunas fases del Pleistoceno.

El área de estudio se sitúa en la llanura aluvial que forma el río Vinalopó en el extremo más meridional de la fosa tectónica de Agost-Monforte, inmediatamente antes de adentrarse en las alineaciones montañosas del anticlinal de la sierra Crevillent - el Colmenar, que la separan de la cuenca de Elche en el Bajo Vinalopó. Estos relieves están formados por calcarenitas y conglomerados de edades comprendidas entre el Mioceno Medio y el Plioceno Superior, que presentan una morfología en cuesta con buzamiento norte de entre 30 y 45 grados, cuyo origen se encuentra en el levantamiento diapírico de las arcillas triásicas del Pantano de Elche. Este movimiento neotectónico origino al norte un sinclinal periférico (rim syncline) con carácter subsidente, franja de hundimiento rellena con los aportes del Vinalopó y sus afluentes, que constituye el área donde se ha centrado la investigación geoarqueológica (Fig. 1a).

A lo largo del Cuaternario el sistema fluvial, no siempre activo, ha dado lugar a un conjunto de terrazas desarrolladas durante extensas fases rexistásicas e individualizadas en breves fases de incisión que han sido puestas en relación con el levantamiento neotectónico del Prebético Valenciano (Cuenca Payá and Walker, 1995). Estos mismos autores propusieron un modelo de sucesión de cuatro niveles de glacis terraza para toda la cuenca desde el Pleistoceno medio, a 120, 75, 35 y 10 m sobre el talweg. Los más altos y antiguos aparecen como retazos de aluvión heterométrico poco cementado del Pleistoceno medio, adosados a las laderas o coronando cerros testigo. Los glacis terraza situados a unos $+35 \mathrm{~m}$ sobre el cauce están formados por sedimentos aluviales, loess, encostramientos calcáreos y paleosuelos hidromorfos del Pleistoceno superior en los llanos y coluviones al pie de los relieves. El glacis terraza a +10 m se compone de una serie de miembros o unidades no siempre superpuestos. La unidad 1, a muro, está constituida por fanglomerados angulosos y paleosuelos calcáreos (calcretas) anteriores al $30 \mathrm{ka}$ BP. La unidad 2 incluye una sucesión microrrítmica de yeso singénico, loess resedimentado y formaciones edáficas de entorno al $20 \mathrm{ka}$ BP. La unidad 3 está formada por aluviones gruesos con clastos de gran tamaño con datación del Tardiglaciar. La unidad 4 es de arenas eólicas, paleosuelos con horizontes gley y pseudogley, y horizontes edáficos húmicos del Holoceno inferior. Finalmente la unidad 5 fue descrita como de canchales con clastos angulosos y poco evolucionados del Holoceno superior. El encajamiento actual de los cauces, no muy intenso, sería posterior a época romana (Cuenca and Walker, 1995, Ferrer García and Blázquez Morilla, 1999).

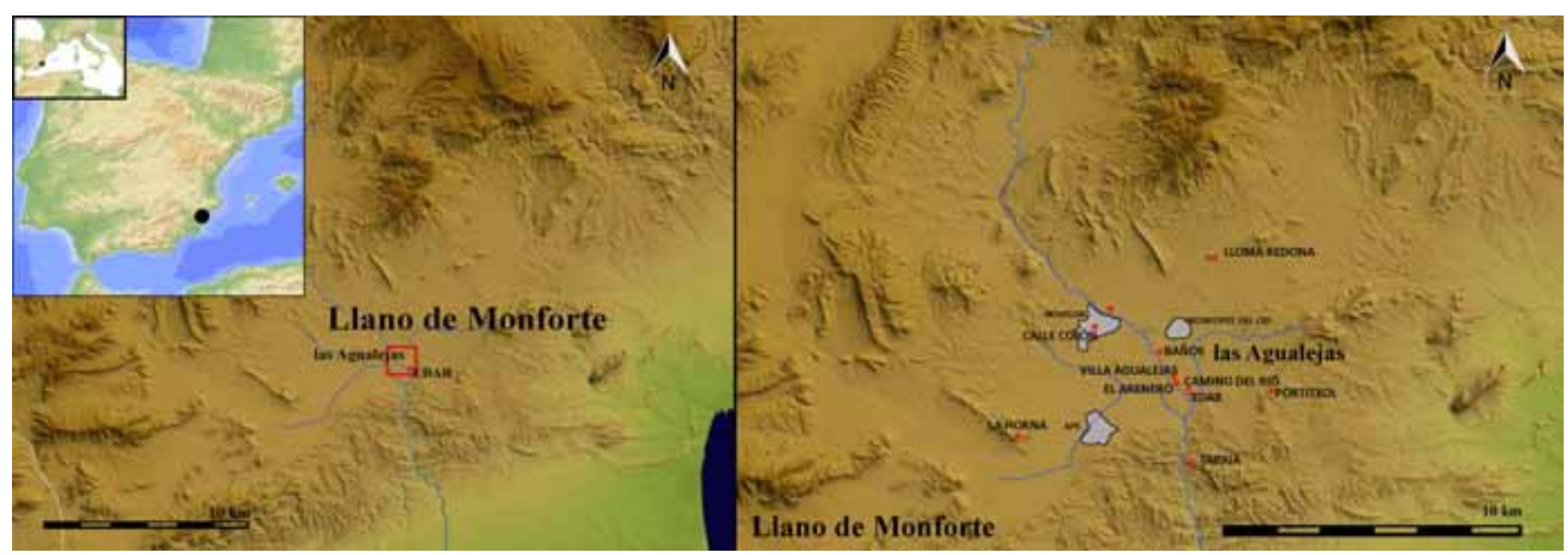

Figura 1. a) Localización del área de estudio. b) Localización de los yacimientos arqueológicos citados en el texto. Base cartográfica del CINSTITUT CARTOGRÀFIC VALENCIÀ 2016.

Figure 1. a) Location of the study area. b) Location of archaeological sites mentioned in the text. CINSTITUT CARTOGRÀFIC VALENCIÀ 2016. 
Ferrer García, C., 2018. Dinámica fluvial durante el inicio del Holoceno superior en el... Boletín Geológico y Minero, 129 (1/2): $305-330$

\section{Ocupación del territorio}

Este espacio geográfico ha tenido una intensa ocupación humana a lo largo de la prehistoria reciente. Se han documentado yacimientos arqueológicos desde el Neolítico antiguo, en torno a hace 7250 años, en la calle Colón y la zona denominada Ledua del casco urbano de Novelda, al noroeste de las Agualejas (Hernández Pérez and Alberola Belda, 1988, García Atiénzar et al., 2006) (Fig. 1b).

La presión humana se intensifica en la Edad de los Metales. Durante el Campaniforme se ocupa el área del yacimiento Camino del Río, en torno a hace 5000 años (Molina Mas and Ortega Pérez, 2010). En la Edad del Bronce Pleno, hace 4000 años, bajo la influencia de la cultura Argárica, se han catalogado más de 22 asentamientos que explotan intensamente los llanos con actividades agropecuarias. Algunos tan relevantes comoTabaià, la Horna, el Portitxol y Lloma Redona (Hernández Pérez, 1983, Jover Maestre and López Padilla, 2004).

El poblamiento fue también relevante desde la protohistoria y hasta al menos época romana. Pero este no fue continuo. La ocupación durante el periodo lbérico pleno fue muy intensa. Se expresó en un área funeraria y ritual de gran extensión, excavada en el Arenero y Camino del Río en las Agualejas, en forma de conjuntos escultóricos monumentales, enterramientos y encachados con restos de ceremonias rituales de entre los siglos VI al IV a. C. (Abad Casal and Alberola Belda, 1990, Abad Casal and Sala Selles, 1992, Abad Casal et al., 1998, Molina Mas and Ortega Pérez, 2010), así como un conjunto de evidencias de asentamientos bajo las villas romanas de las Agualejas y Baños, en forma de restos asociados a espacios marginales al hábitat (Molina Mas, 2015).

Los restos arqueológicos fechados entre el siglo III y I a. C. son escasos. Se ha propuesto un posible despoblamiento del Bajo y Medio Vinalopó (Tendero Porras et al., 2014, Molina Vidal, 2002). Con todo, se mantiene un uso testimonial del área sacra. Destaca un único enterramiento en las Agualejas, con un ritual poco ortodoxo (Moratalla, 2005) y las estructuras rituales excavadas en los yacimientos de EDAR y Camino del Río (Segura et al., 2012, Molina Mas and Ortega Pérez, 2010).

En el cambio de Era se han propuesto transformaciones importantes en la estructura del poblamiento. Una reorganización del territorio y su explotación plena según el modelo romano se habría producido en el siglo inmediatamente siguiente, en relación con la centuriación del área (Moratalla, 2001), que se hallaba bajo el control de la colonia romana de llici (Grau Mira and Moratalla Jávega, 1997 y 2001, Molina
Mas, 2015), que se expresa aquí con la construcción de la villa de los Baños (Ortega Pérez et al., 2008). A lo largo del siglo II d. C. se habría mantenido la presión sobre el medio (Márquez, 2006).

Finalmente, en época tardorromana la ocupación de este territorio con asentamientos rurales, que sigue ligado culturalmente con el Bajo Vinalopó y la ciudad de Ilici, parece haber sido muy intensa, en particular a partir del siglo IV d. C. (Tendero Porras et al., 2014). En la segunda mitad del siglo III d. C. se construye la villa de las Agualejas, que alcanza su mayor esplendor en el siglo IV d. C., momento en el que se amplía la villa de Baños (Ortega Pérez et al., 2008, Molina Mas, 2015).

\section{Metodología}

Los registros sedimentarios han sido estudiados con métodos geomorfológicos, estratigráficos y sedimentológicos. El establecimiento de su evolución en el tiempo, la cronoestratigrafía, se basa en su datación relativa a partir de la información contextual que ofrece el registro arqueológico.

La correlación de los datos paleoambientales en este contexto geográfico con la evolución del poblamiento y la gestión del territorio de las sociedades productoras antiguas, nos permite abordar la problemática de la interacción entre medio y comunidades humanas, en especial la derivada del impacto de las actividades humanas en el sistema fluvial, cuestión genuinamente geoarqueológica y relevante para la sociedad contemporánea, en relación con los problemas ambientales actuales.

Los datos geomorfológicos proceden del reconocimiento de las formas del paisaje, de los ambientes sedimentarios y de perfiles estratigráficos, a través del trabajo de campo y la fotointerpretación de pares esteroscópicos de fotografía aérea de mediados de los años 50 del siglo pasado (aproximadamente a escala 1:33.000) y de otros vuelos más recientes (a escalas diversas, 1:25.000 ó 1:18.000), además del análisis en gabinete de diversos documentos cartográficos, topográficos y geológicos.

El trabajo estratigráfico ha consistido en el análisis completo de los perfiles sedimentarios disponibles, la determinación de las discontinuidades verticales y horizontales y la definición de las unidades o niveles que los conforman, a partir de criterios observables de visu, tales como la composición del sedimento, el color (con el apoyo de la tabla Munsell Soil Color Charts, 2000), la estructura interna y el grado de cohesión. El resultado son perfiles litoestratigráficos que interpretamos en nuestro trabajo como perfiles 
tipo, columnas representativas de los procesos que caracterizan las secuencias fluviales que en el caso del yacimiento arqueológico de EDAR fueron muestreados para su análisis sedimentológico.

La caracterización sedimentológica de los depósitos de EDAR ha consistido en el estudio de sus rasgos litológicos y texturales. El análisis granulométrico está considerado como una buena herramienta para la caracterización de los sedimentos, la identificación y definición de los agentes y procesos deposicionales $y$, en consecuencia, para la caracterización de las condiciones ambientales que han actuado en la formación de los depósitos (Shackley, 1975, Reineck and Singh, 1980, Hartmann and Flemming, 2007). La técnica aplicada para determinar el tamaño del grano ha sido el tamizado en seco para la fracción gruesa, por encima de $2 \mathrm{~mm}$, y el empleo del método de la pipe- ta de Robinson para la fracción fina, descritos de manera conjunta como de tamiz/pipeta (sieve-pipette) o de la pipeta simplemente. La posibilidad de comparar resultados que ofrece este método, su consolidación, su bajo coste y la aceptable exactitud, la hacen la opción óptima para este estudio. En concreto la metodología aplicada ha sido la propuesta y desarrollada en el Departament de Geografia de la Universitat de València (Fumanal, 1986), que sigue el modelo estándar descrito en diversos manuales (Soil Survey Staff, 2014, e.g.). Los datos derivados del análisis textural de la fracción fina han sido representados en histogramas de frecuencias, en curvas acumulativas y curvas semiprobabilísticas con apoyo del software Microsoft Excel ${ }^{\circ}$ y se han calculado parámetros estadísticos según el modelo de Folk y Ward (1957) con el programa Gradistat ${ }^{\odot}$ versión 8 (Blott and

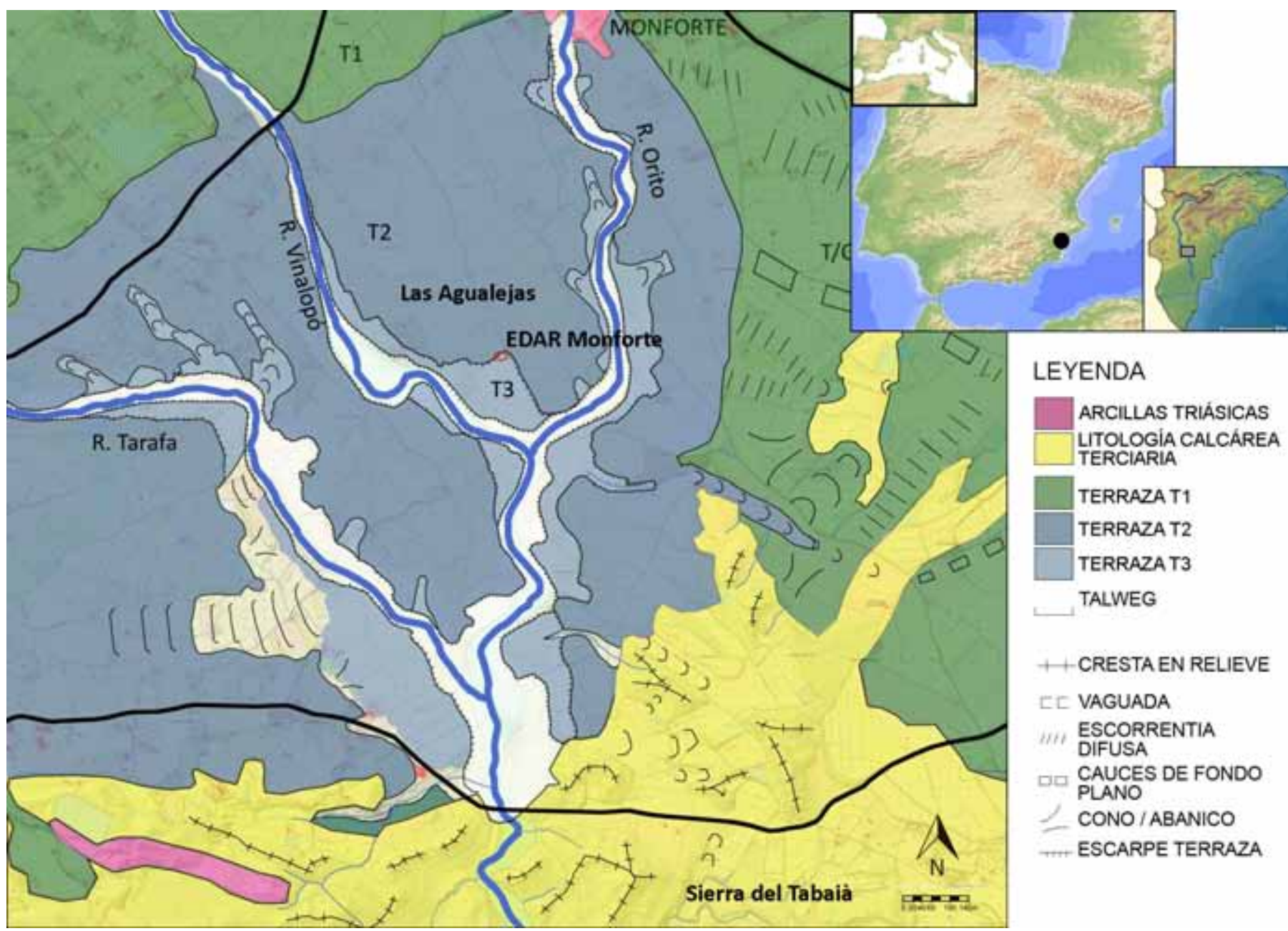

Figura 2. Esquema geomorfológico con los niveles de terraza fluviales en la zona de estudio de las Agualejas del curso medio del río Vinalopó.

Figure 2. Geomorphological scheme with river terrace levels in las Agualejas (Vinalopó middle watercause). 
Pye, 2001 y 2012). Para la interpretación de las representaciones gráficas y los parámetros nos hemos apoyado en los trabajos de Folk y Ward (1957), Tricart (1965), Visher (1969), Reineck and Singh (1980), Fumanal (1986), Pye (1987), Flemming (2007), Boggs (2009) y Mycielska-Dowgiałło and LudwikowskaK dzia (2011), entre otros.

El análisis de los aspectos morfológicos de la fracción por encima de 0,062 $\mathrm{mm}$ se basa en estudios de visu; en el caso de la fracción arenosa hasta los 0,5 $\mathrm{mm}$ se llevó a cabo con el apoyo de la lupa binocular (Fumanal, 1986). También se ha contemplado el análisis del porcentaje de carbonato cálcico y de materia orgánica. Su valoración se ha hecho en el primer caso mediante el ataque con $\mathrm{HCl}$ de 10 gramos de muestra de fracción fina. La materia orgánica se ha calculado a través del método analítico de oxidación del carbo- no orgánico con un oxidante, el dicromato potásico (Walkley and Black, 1934).

\section{Resultados \\ Los niveles de glacis terraza recientes de las Agualejas}

El estudio estratigráfico detallado nos ha permitido diferenciar tres niveles de glacis terraza correlacionables en parte con el modelo propuesto por Cuenca Payá y Walker (Fig. 2). La ubicación de los perfiles y los cortes se hallan en la figura 3. Prestaremos especial atención al techo de la terraza T2 y a la totalidad de la terraza T3, ya que tienen su desarrollo a lo largo del Holoceno superior.

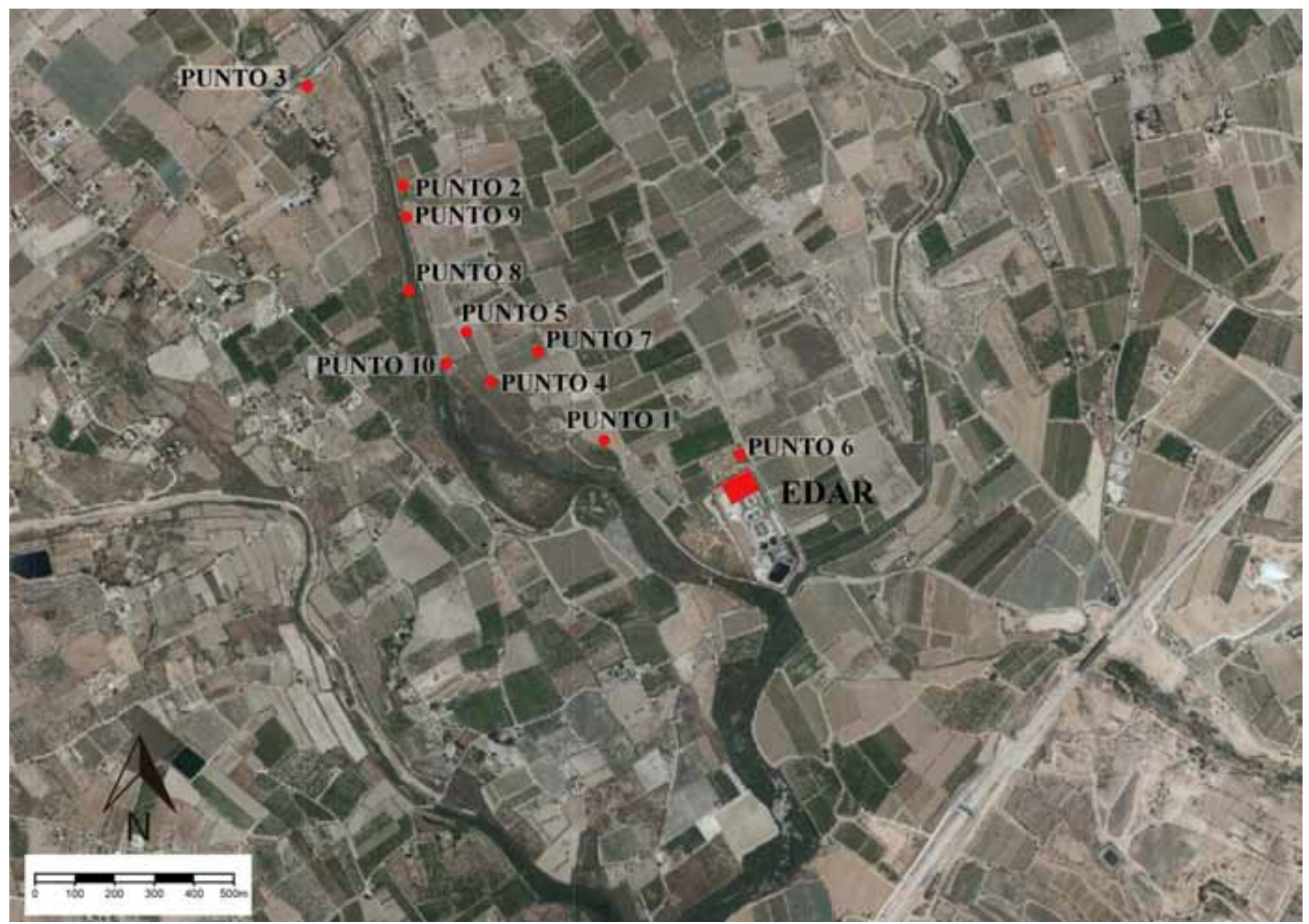

Figura 3. Localización de los puntos citados en el texto y del yacimiento de EDAR. Ortofoto base del CINSTITUT CARTOGRÀFIC VALENCIÀ 2016.

Figure 3. Location of the points mentioned in the text and the archaeological site studied. Ortophoto base CINSTITUT CARTOGRÀFIC VALENCIÀ 2016. 
Ferrer García, C., 2018. Dinámica fluvial durante el inicio del Holoceno superior en el... Boletín Geológico y Minero, 129 (1/2): $305-330$

La terraza $T 1$, la superior, en torno a $+10 \mathrm{~m}$ por encima del cauce, se conserva parcialmente en la parte alta del área, en forma de retazos aislados por pequeños escarpes. Los perfiles están constituidos por estratos laminares de limoarcillas de color gris verde claro (GLEY1 8/1) de 20 a $50 \mathrm{~cm}$ de potencia, que alternan con otros similares pero consolidados de en torno a los $15 \mathrm{~cm}$ de grosor (punto 1 de la figura 3). Sus rasgos coinciden parcialmente con los del miembro basal 2 de la terraza A del Pleistoceno superior final del modelo de Cuenca Payá y Walker (1995). Serían expresión de una fase de drenaje impedido en lo que hoy es la cuenca media del río, con condiciones ambientales algo más húmedas que las actuales. Fumanal y Carmona (1995) dataron formaciones edáficas pleistocenas en torno al $22 \mathrm{ka}$ BP por TL en el área valenciana que podrían ser coincidentes con esta fase.

LaTerrazaT2 ocupa gran parte de la zona por debajo de la cota $208 \mathrm{~m}$ de altitud, a unos $+8 /+7 \mathrm{~m}$ por encima del cauce del río. Está constituida por dos extensas unidades sedimentarias bien diferenciadas en los perfiles fluviales estudiados. La basal, de facies braided, incluye pasadas horizontales de fracción gruesa rodada heterométrica y matriz arenosa de color marrón muy pálido (10YR 8/2). La superior es de limos y arcillas de color marrón pálido (10YR 6/3) de llano de inundación, con pasadas horizontales decimétricas ligeramente cementadas y lentejones de fracción gruesa, también heterométrica, imbricada a favor de la corriente (Figs. 4a y b). Fuera del contexto del escarpe fluvial se han documentado sobre la superficie de esta terraza niveles arenosos de color blanco (10YR 8/1) a los que se superponen limoarcillas de color gris que interpretamos como horizontes gley, o las propias arenas con colores amarrillo marrón (10YR 6/8) y blanco gris (GLEY1 8/1), afectadas por procesos de hidromorfía pseudogley (punto 3 de la figura 4c).

Según el modelo de Cuenca Payá y Walker (1995) las unidades basales de esta terraza, con facies braided, se correspondería con el miembro 3, formado durante el Tardiglaciar. Las formaciones arenosas y edáficas superpuestas se corresponderían con el miembro 4 del modelo, que tendría una datación del Holoceno inferior, establecida a partir de sendas fechas de entre el 10 y el 9 ka BP obtenidas para un suelo enmarcado por niveles eólicos en el pantano de Elda y para una calcreta sobre un manto eólico en la confluencia de la rambla Caprala con el río Vinalopó (Cuenca Payá y Walker, 1995). En coherencia con este modelo se han documentado formaciones edáficas datadas entre el Holoceno inferior y medio sobre arenas eólicas en la cuenca alta del Vinalopó (Fernández
López de Pablo et al., 2011, Fernández-López de Pablo et al., 2014).

El techo de la terraza T2 es de particular interés en nuestro trabajo. En los puntos 3, 4 y 5 se han identificado sucesivos perfiles en los que las arenas con manchas ocres de hidromorfía poseen a techo un contacto neto erosivo, con formaciones sedimentarias de carácter aluvial, formadas por limos y arcillas de color marrón pálido (10YR 7/3) con estructuras poligonales, que incluye en toda su potencia abundantes fragmentos cerámicos de los periodos Ibérico pleno, lbero-romano y Romano. En el punto 6, en el yacimiento arqueológico del Camino del Río, se documentó sobre las arenas y bajo la superficie de contacto con las limoarcillas, que buzan hacia el cauce, un conjunto de estructuras negativas, silos del Calcolítico, del 3000 a. C., un nivel de enterramientos de los siglos V y IV a. C. y una balsa del siglo II a. C. (Molina Mas and Ortega Pérez, 2010). Por su parte, la excavación en la villa romana de las Agualejas (punto 7 de la figura 3) mostró la formación sobre la superficie de erosión de un depósito de $50 \mathrm{~cm}$ de potencia de sedimentos que se describen como de aluvión, de nuevo limoarcillas de color marrón muy pálido (10YR $7 / 4$ ), sellado por las estructuras de la villa de los siglos III y IV d. C. (Molina Mas and Ortega Pérez, 2010).

La terraza T3 aparece por debajo de la cota $200 \mathrm{~m}$ de altitud y se sitúa a unos $+6 /+5 \mathrm{~m}$ sobre el talweg del Vinalopó. En la parte alta se preserva parcialmente junto al cauce. En la confluencia del río Vinalopó con la rambla del Orito, a partir de la cota 198 m, conforma la llanura (Fig. 2). Las características estratigráficas de esta terraza se han observado en los perfiles abiertos en el área de estudio de $1 \mathrm{~km}^{2}$. Se presentan tres perfiles que sintetizan sus rasgos, fundamentalmente diversas facies braided que culminan a techo con una unidad bastante homogénea de limos de llano de inundación, y que pueden concretarse en cuatro unidades diferenciadas.

El perfil 1 aparece al descubierto en un pequeño tramo aislado de terraza (Punto 8 de la figura 3), erosionado a techo por la construcción de un camino. Incluye una formación sedimentaria, denominada unidad 1, constituida por $170 \mathrm{~cm}$ de bloques y cantos rodados con arena gruesa de color marrón muy pálido (10YR 8/2), formando pasadas horizontales más o menos homométricas, con cierto grado de imbricación, así como pasadas de fracciones más finas, gravas y arenas gruesas con apenas matriz también en disposición horizontal e imbricación (Fig. 5a). A cota $+150 \mathrm{~cm}$ respecto al talweg se encontró un único diente de hoz en sílex que ha sido datado de la Edad del Bronce o fase cultural posterior. 

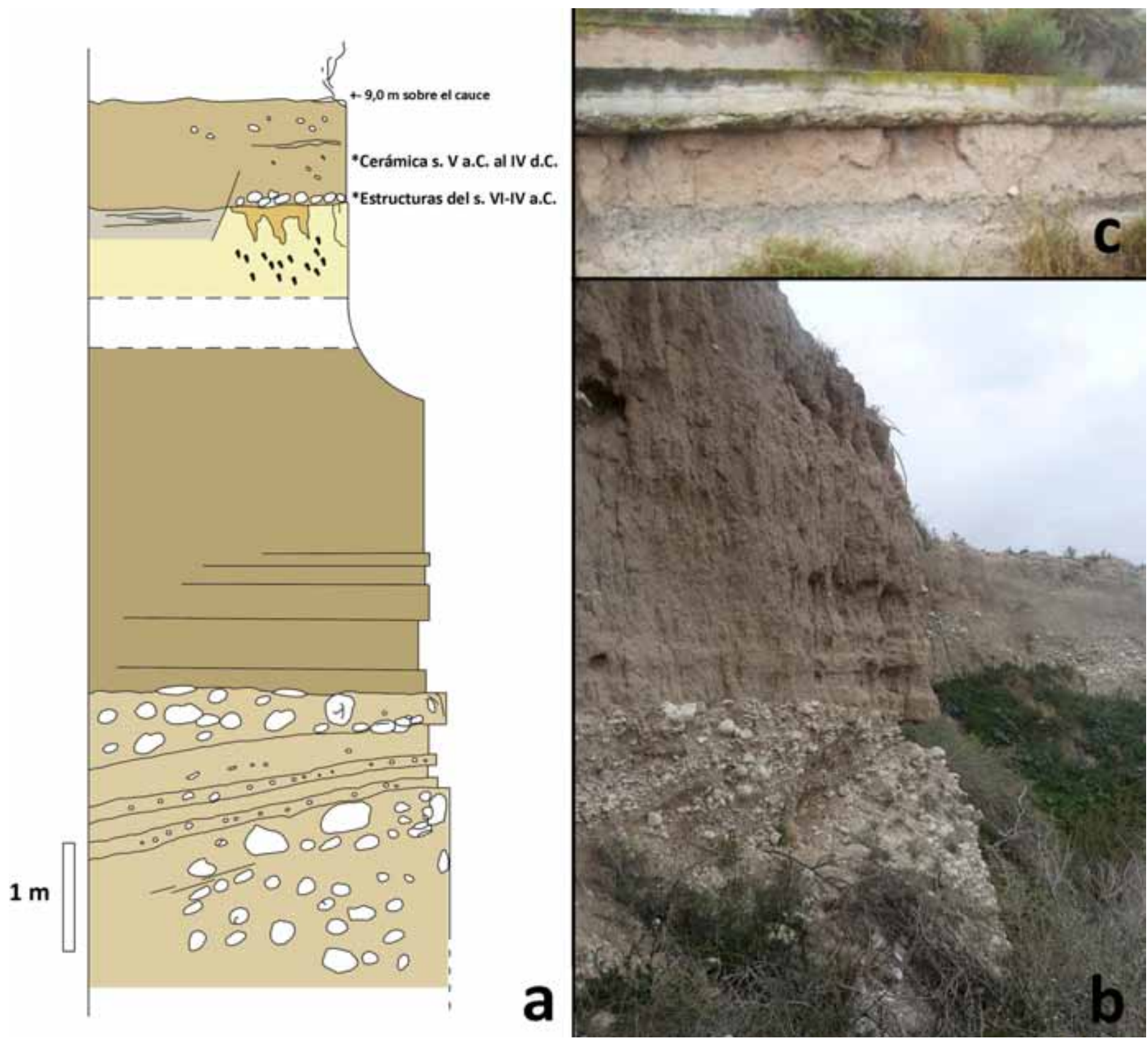

Figura 4. Terraza T2: a) Perfil tipo de la terraza. b) Unidades estratigráficas de la terraza en escarpe. c) Arenas y horizontes edáficos gley a techo de la terraza.

Figure 4. Terrace T2: a) Synthetic profile. b) Stratigraphic units of the terrace; c) Sands and gley soil horizons up to the terrace roof.

El perfil 2 (Punto 9 de figura 3) está constituido por cuatro unidades con contactos progresivos (Fig. $5 \mathrm{~b}$ ). La unidad 1, de $110 \mathrm{~cm}$ de potencia vista, está formada por pasadas de bloques y cantos rodados heterométricos empastados en matriz arenosa de color marrón muy pálido (10YR 8/2). A base forma laminaciones oblicuas con imbricación y a techo presenta estructura masiva y una pasada de cantos horizontales también imbricados. Le sigue la unidad 2, forma- da a muro por un estrato de $80 \mathrm{~cm}$ de gravas y cantos de menos de $3 \mathrm{~cm}$ con muy escasa matriz arenosa de color similar (10YR 7/3) y estructura masiva, aunque se observa el predominio de la disposición horizontal y puntuales signos de imbricación, y a techo por un estrato de cantos masivos de mayor tamaño (de menos de $6 \mathrm{~cm}$ ). La unidad 3 está constituida por un estrato de $45 \mathrm{~cm}$ de bloques y grandes cantos rodados y heterométricos con escasa matriz de limos $y$ 


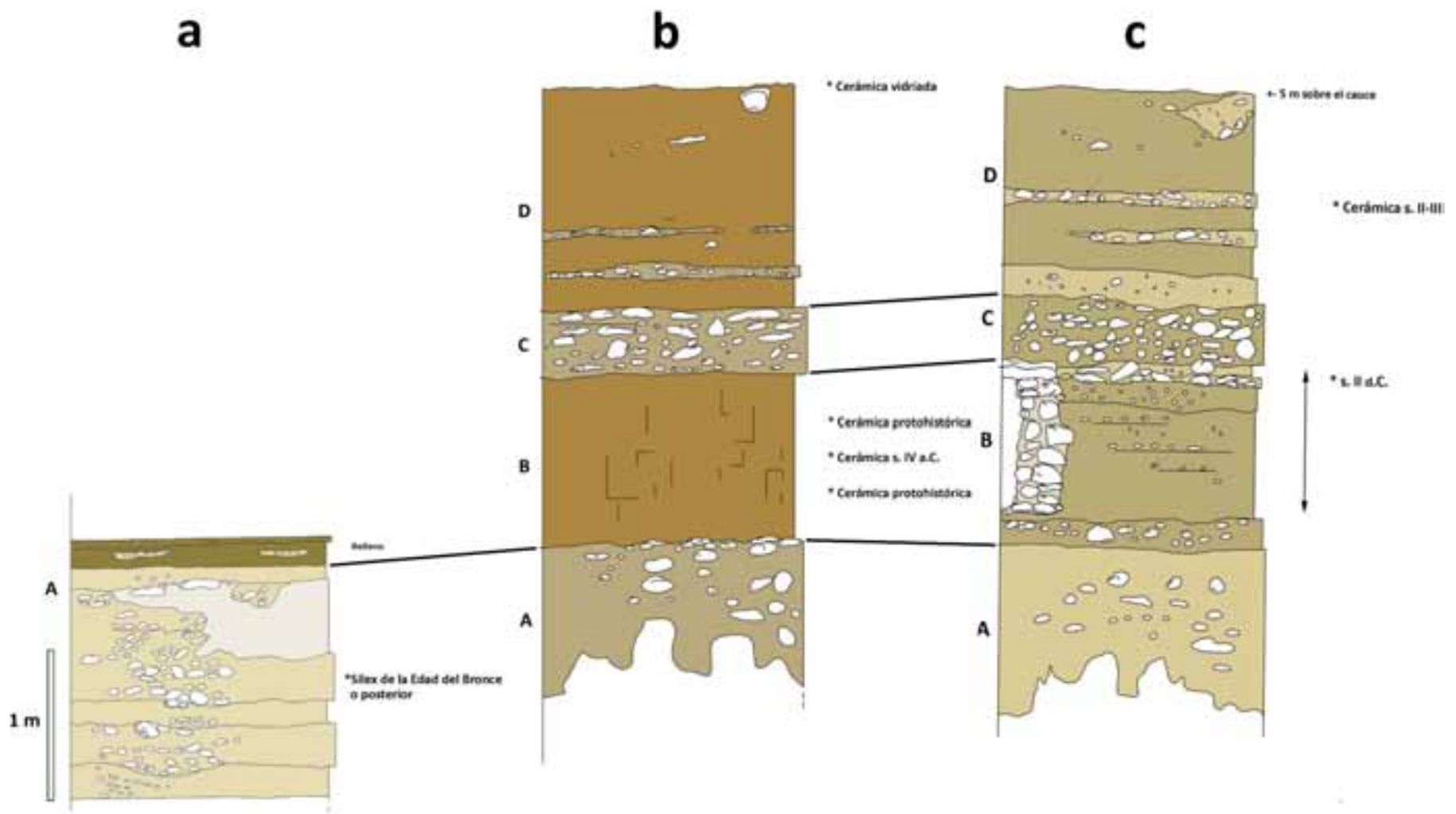

Figura 5. Terraza T3: a) Perfil en el punto 8. b) Perfil en el punto 9. c) Perfil en el punto 10. Figure 5. Terrace T3: a) Profile at point 8. b) Profile in point. c) Profile at point 10.

arenas de color idéntico al de la anterior unidad. La fracción gruesa presenta en algunos casos imbricación, aunque predomina la distribución masiva y la tendencia a la horizontalidad. Se intercalan capas de gravas y lentejones de arenas gruesas con laminaciones horizontales. Culmina esta unidad un estrato de $15 \mathrm{~cm}$ de gravas y cantos pequeños con estructura masiva sin apenas imbricación. La unidad 4 es de fracción fina y color también marrón muy pálido (10YR 7/3), con $100 \mathrm{~cm}$ de potencia. En ella se intercalan algunos cantos aislados con disposición no siempre horizontal, además de lentejones de bloques, cantos y gravas rodados con matriz similar y estructura masiva.

El perfil 3 (Punto 10 de la figura 3) está constituido por cuatro unidades con contactos netos no erosivos (Fig. 5c). La basal, unidad 1, de $100 \mathrm{~cm}$ de potencia, está formada por pasadas de bloques y cantos rodados heterométricos empastados en matriz arenas de color marrón muy pálido (10YR 7/3) y estructura masiva. La unidad 2, de igual potencia y color, está constituida por arcillas, limos y arenas, que forman estructuras poligonales, aunque se identifican algunas pasadas horizontales más arenosas. La unidad 3, de hasta $40 \mathrm{~cm}$ de potencia, está formada por cantos y gravas rodados heterométricos con disposición horizontal a muro y masiva, sin imbricación, con matriz arcillosa de color marrón muy pálido (10YR 7/3). La unidad 4 es de nuevo de arcillas, limos y arenas formando estructuras poligonales, de igual color y 130 $\mathrm{cm}$ de potencia. En la base presenta un estrato lenticular de gravas rodadas con matriz arenosa y disposición horizontal sin imbricación.

La unidad 2 incluye en toda su potencia fragmentos cerámicos a torno. Se encontraron a 20,50 y 80 $\mathrm{cm}$ de su techo. Uno de ellos, de la parte más alta, se corresponde con una cerámica decorada por las dos caras del siglo IV a. C. A techo de la terraza se documentan de nuevo cerámicas vidriadas medievales.

En el perfil se descubrió una conducción o acequia del siglo II d. C. Está construida sobre la unidad 2, profundizando en ella hasta $80 \mathrm{~cm}$ con paredes forradas con bloques rectangulares con mortero. Su techo se halla bajo la base de la unidad 3 y está formado por un enlosado que indica que se construyó desde el nivel del suelo. Por otro lado, en uno de los lentejones de la unidad 4 se encontraron fragmentos de cerámicas del siglo II-III d. C. a unos $70 \mathrm{~cm}$ del techo de esta unidad. Sobre la terraza se documentaron cerámicas vidriadas medievales. 
Ferrer García, C., 2018. Dinámica fluvial durante el inicio del Holoceno superior en el... Boletín Geológico y Minero, 129 (1/2): $305-330$

Es posible establecer la correlación estratigráfica de las unidades que conforman los perfiles estudiados en la terraza T3. La base conocida está constituida por la unidad 1 de barras longitudinales de gravas y cantos y facies de fondo de canal, con al menos un resto arqueológico de la Edad del Bronce o posterior. En la que se ha descrito como unidad 2, en la que alternan las facies de barras longitudinales de gravas y cantos o depósitos de revestimiento de canal, con depósitos de overbank e inundación, se ha constatado la existencia en su parte superior de abundantes restos cerámicos que podrían adscribirse al siglo IV a. C. en adelante. Su techo incluye una estructura de conducción de agua del siglo II d. C., que podría indicar una estabilización en el sistema y una ralentización de los procesos de acreción de la terraza. La unidad 3 de la terraza se corresponde con lo que parece ser un potente y generalizado depósito de debris flow, en ocasiones de fondo de canal, que se habría producido con posterioridad al siglo II d. C. En el extremo superior de la terraza, unidad 4, predominan las facies de llano de inundación, aunque se repiten las coladas y facies de fondo de canal de manera más puntual. La cerámica encontrada a media altura de este segmento nos aporta una datación del siglo II a III d. C., que constituiría una referencia cronológica para la formación de la unidad previa.

\section{El yacimiento arqueológico de EDAR}

El yacimiento arqueológico de EDAR se halla en la partida de las Agualejas formando parte de la unidad 2 de la terraza T3. Se ubica a $197 \mathrm{~m}$ de altitud en un interfluvio en las proximidades de los cauces encajados (unos $4 \mathrm{~m}$ ) del río Vinalopó y de la rambla de EI Orito, en una de las partes más bajas del llano de Monforte (Figs. 2 y 3 ).

El conjunto arqueológico principal recuperado en la excavación está formado por los restos de un edificio o espacio construido del siglo I a. C., del cual se conserva el fundamento de tres de sus muros (U.E. 1010). Su espacio interno, de 13,3 por $7,8 \mathrm{~m}$ y planta rectangular, presenta la particularidad de incluir una estructura negativa, un rebaje del nivel basal en forma de concavidad que ocupa su flanco occidental (de 4,9 m de Este a Oeste y 6,25 m de Norte a Sur). Se abre en un nivel o estrato de arcillas y limos de color gris (U.E. 115), está relleno de sedimentos similares con abundantes restos arqueológico (U.E. 114) y fue sellado con una extensa acumulación de bloques y cantos rodados procedentes de los muros de la estructura arqueológica que la delimita (Fig. 6). El extenso repertorio de materiales que se recuperó en la excavación del relleno de la cubeta permite deducir el desarrollo de prácticas rituales relacionadas con el agua coincidiendo con su progresiva amortización entre el 90 y el 40 a. C. Ceremonias de las que forman parte el consumo de alimentos y el depósito de recipientes cerámicos.

Durante el proceso de excavación del yacimiento de EDAR se llevó a cabo el estudio estratigráfico de cuatro perfiles en relación con la estructura arqueológica descrita. Se trata del sustrato de sus muros perimetrales, de un testigo sedimentario central, coincidente con la U.E. 114, y de unos niveles de arenas y limoarcillas posteriores a su amortización (Figs. 7a, b y c).

El nivel I, basal, está formado por arenas masivas de color marrón muy pálido (10YR 7/4) con algunas pasadas de gravas rodadas, y una potencia vista máxima de $100 \mathrm{~cm}$. Presenta manchas de color ocre (10YR 6/6) a techo en el perfil 1 y motas de color ocre y gris oscuro en el perfil 2, que parecen revelar la precipitación de hierro oxidado y manganeso. En el perfil 5 está constituido por arenas de color marrón muy pálido (10YR 8/2) con estratigrafía laminar horizontal, que en el perfil abierto, de $30 \mathrm{~cm}$ vistos, incluye un total de 10 capas de entre 2,5 y $6 \mathrm{~cm}$ de potencia, alternando arenas finas con estratificación laminar suavemente ondulada, en ocasiones cruzada de bajo ángulo, con otras de arena gruesa con estructura interna grano-creciente. Se observan además en este perfil algunas estructuras de ripple foreset. El nivel incluye restos cerámicos del siglo III-I a. C. y constituye en el último perfil la base del muro construido a inicios del siglo I a. C., posiblemente hacia las décadas del 100-80 a. C.

En la distribución textural de las muestras analizadas se identifican dos claros segmentos superpuestos, que revelan la existencia de flujos de diferente energía transportando la fracción en saltación con capacidad de clasificación, y que pudiera asociarse a medios fluviales arenosos (Visher, 1969), pero también a medios eólicos (Mycielska-Dowgiałło and Ludwikowska-kedzia, 2011) (Fig. 8). El análisis morfoscópico de las arenas muestra que se trata fundamentalmente de cuarzo transparente redondeado y mate (entre el 55 y el $50 \%$ ), resultado de procesos eólicos, aunque se describe un $35-30 \%$ de cuarzos briIlantes angulosos. El resto son calizas blancas, beige u ocres rodadas. Así, aunque los rasgos texturales de estas muestras no permiten una interpretación del todo concluyente acerca de su origen, sus características morfoscópicas, y en especial las estratigráficas, revelan que son el resultado de la dinámica sedimentaria fluvial que procesa sedimento de origen eólico. La presencia de carbonato cálcico es muy baja, el 


\section{Sección A}

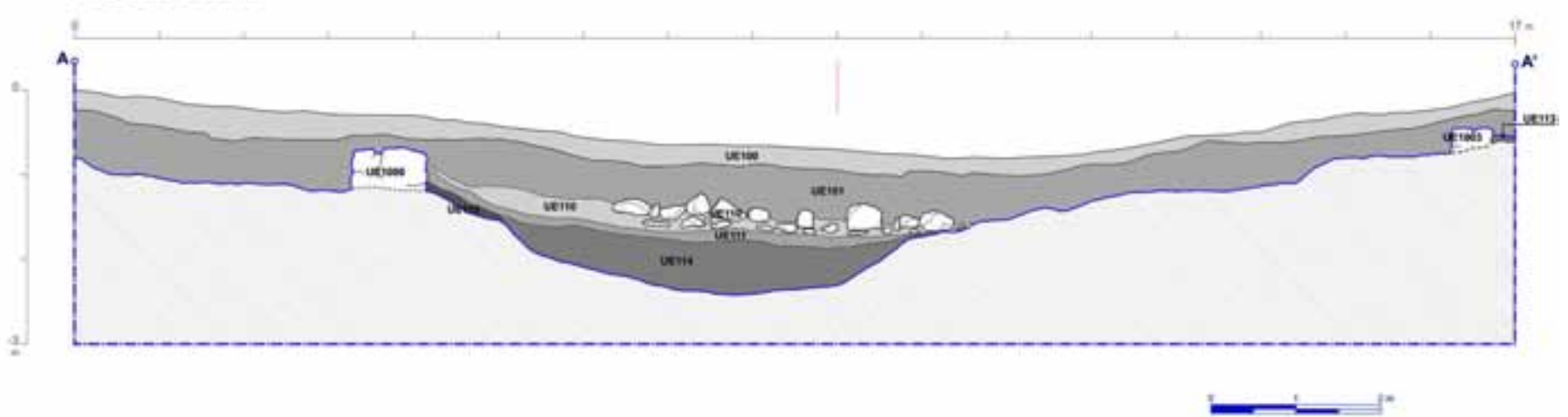

\section{Sección B}
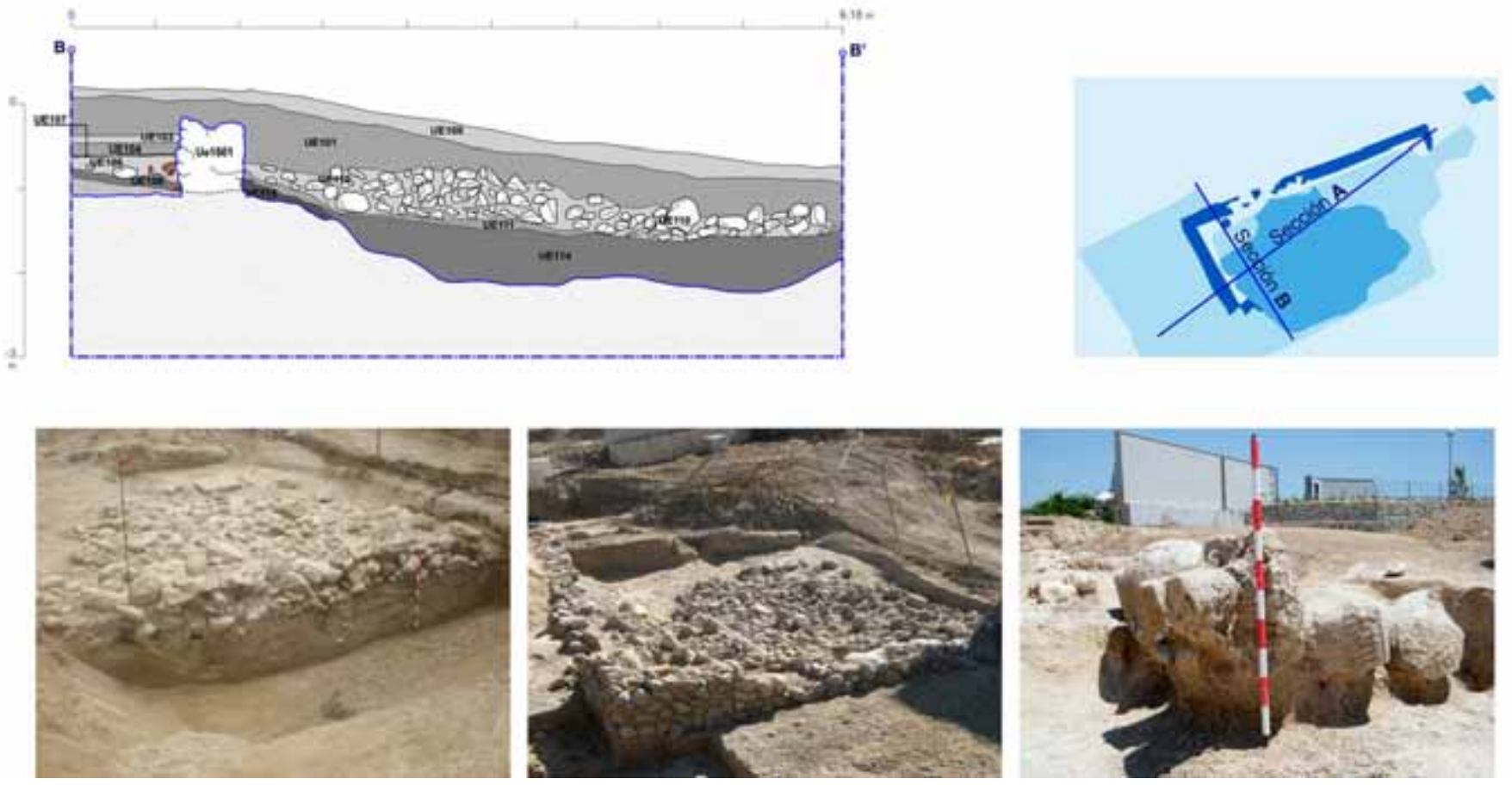

Figura 6. Perfiles estratigráficos del yacimiento arqueológico EDAR. Fotografías de la cubeta rellena de sedimentos en contexto arqueológico y la acumulación de cantos y bloques que la sellan (ver referencias en el texto). Planimetría de Arquealia S.L.

Figure 6. Stratigraphic profiles of the EDAR archaeological site. Photos of the tank filled with sediments in the archaeological context and the accumulation of cobbles and boulders that sealed the structure (see references in the text). Property of Arquealia S.L.

$20 \%$, así como el porcentaje de materia orgánica, en torno al $0,1 \%$, excepto en el perfil 5 , en el que llega al $0,35 \%$, tal vez en relación con la presencia de actividades humanas, ya que aquí este nivel sustenta las estructuras del s. I a. C. (Tabla 1).

El nivel II, en contacto gradual con el inferior, es un estrato de limos y arcillas de hasta $40 \mathrm{~cm}$ de potencia, documentado en los perfiles 1, 2 y 3 . En el perfil 1 forma agregados resistentes de color gris claro (10YR $7 / 2$ ) con moteado de color ocre (10YR 6/6) y negro de distribución irregular, y diferentes formas y límites. En los perfiles 2 y 3 es en cambio de color gris homogéneo y presenta formas poligonales y grietas de retracción. Se identifica con la U.E. 115, con materiales arqueológicos que permiten ubicar su formación en torno a los siglos II-I a. C, como mínimo desde el 160-150 a. C., pero se podrían remontar al 180 a. C. Sustenta el mismo muro que el anterior nivel en el perfil 5, como ya vimos, construido en torno al inicio del siglo I a. C. 

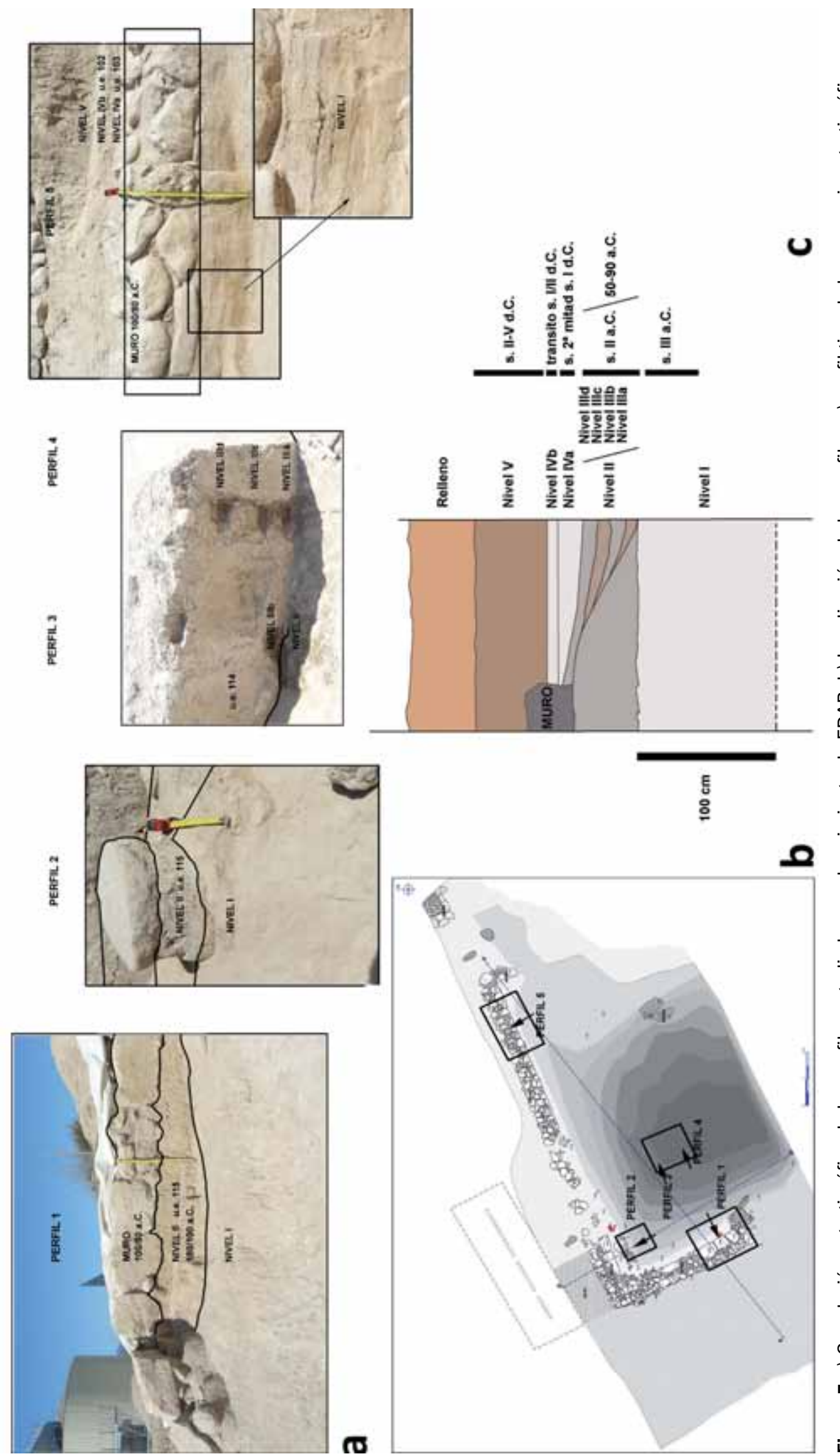

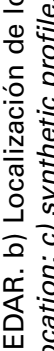

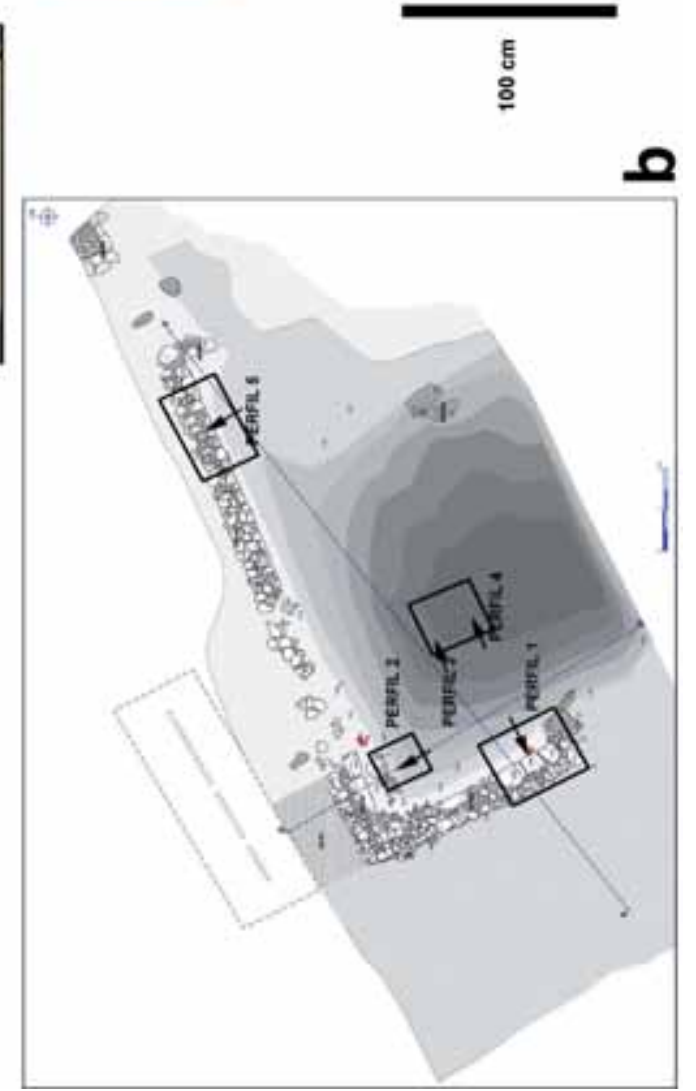

엉

웡

$\frac{2}{2}$

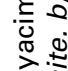

के के

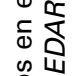

원

웅

कृ

里

बू

은ㅎㅇ

응

준 $\frac{1}{4}$

흄ำ

क्ष

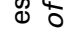

:등

$\frac{\pi}{2} \frac{\pi}{2}$

ठั

$\bar{\pi} \pi$

กำ

繥 
Ferrer García, C., 2018. Dinámica fluvial durante el inicio del Holoceno superior en el... Boletín Geológico y Minero, 129 (1/2): $305-330$

\begin{tabular}{|l|l|l|l|l|l|l|l|l|l|}
\hline Nivel & Arena & Limos & Arcilla & M.O. & Carb. & Media & Clasif. & Asimet. & Kurtos. \\
\hline P1-I & 84,26 & 6,8 & 8,94 & 0,14 & 19,24 & 2,581 & 1,851 & 0,599 \\
\hline P1-II & 25,94 & 18,8 & 55,26 & 0,59 & 41,05 & 7,016 & 2,93 & $-0,63$ \\
\hline P2-I & 80,87 & 11,79 & 7,34 & 0,06 & 25,14 & 3,033 & 2,085 & 0,65 \\
\hline P2-II & 17,04 & 21,87 & 61,09 & 0,5 & 40,61 & 7,478 & 26,58 & $-0,735$ \\
\hline P3-II & 22,04 & 28,2 & 49,76 & 0,54 & 45,27 & 7,14 & 2,562 & $-0,435$ \\
\hline P3-IIIb & 8,76 & 26,53 & 64,71 & 0,29 & 41,98 & 8,355 & 1,919 & $-0,683$ & 1,084 \\
\hline P4-IIIa & 19,52 & 20,43 & 60,05 & 0,43 & 46,85 & 7,399 & 2,661 & $-0,68$ \\
\hline P4-IIIc & 48,62 & 13,04 & 38,34 & 0,43 & 41,25 & 5,45 & 3,201 & 0,218 \\
\hline P4-IIId & 42,96 & 12,48 & 44,56 & 0,43 & 40,27 & 6,349 & 3,134 & $-0,349$ \\
\hline P5-I & 82,9 & 4,98 & 12,12 & 0,35 & 19,88 & 3,772 & 2,233 & 0,6 \\
\hline P5-IVa & 82,68 & 9,53 & 7,79 & 0,06 & 22,74 & 3,089 & 1,977 & 0,54 \\
\hline P5-IVb & 87,97 & 5,75 & 6,28 & 0,09 & 23,49 & 2,246 & 1,708 & 1,613 \\
\hline P5-V & 45,15 & 14,21 & 40,64 & 0,36 & 48,01 & 5,839 & 3,12 & $-0,014$ \\
\hline
\end{tabular}

Tabla 1. Datos sedimentológicos de los niveles estudiados en EDAR, porcentajes de arena (sand), limo (silt), arcilla (clay), materia orgánica (O.M.) y carbonato cálcico (Carb.); parámetros estadísticos (Folk and Ward, 1957): tamaño medio (mean), clasificación (sorting), asimetría (skew.) y kurtosis (kurt.).

Table 1. Sedimentological data of EDAR archaeological site levels, percentage of sand, silt and clay, percentages of organic matter (O.M.) and calcium carbonate (Carb.); statistical parameters of grain size (Folk and Ward, 1957): mean, sorting, skewness and kurtosis.

La distribución textural muestra un segmento relevante en la fracción fina (por encima del $35 \%$ ) que denota la presencia de procesos de decantación. La curva acumulativa es siempre hiperbólica y los parámetros gráficos muestran un tamaño medio en fracción limo fino, muy baja clasificación, platocurtosis y asimetría negativa muy marcada; rasgo este último que podría estar en relación con la disminución de la energía del medio (McLaren and Bowles, 1985). La curva semiprobabilística presenta dos segmentos de moderada pendiente que pudiera asociarse a medios de elevada densidad (Visher, 1969), pero se acerca, por la pendiente de en torno a los 45 grados del segmento en saltación, a un modelo descrito como de llano de inundación (Mycielska-Dowgiałło and Ludwikowska-kedzia, 2011).

El porcentaje de materia orgánica se sitúa entre el 0,5 y el $0,6 \%$, que podemos considerar tanto en relación con la presencia humana como con discretos procesos de edafización. También se incrementa el porcentaje de carbonatos (entre el 40 y el $45 \%$ ). La alternancia de color revela procesos de hidromorfía edáfica, básicamente procesos de reducción del manganeso y el hierro (gris verdoso o azulado), alternando con procesos de oxidación (anaranjado u ocre en el hierro, negro en el manganeso).

El nivel III conforma una serie de subunidades registradas en sendos perfiles abiertos en un testigo del interior de la estructura definida por los muros del edificio. Se trata de la U.E. 114, relleno que amortiza la pequeña depresión central, formado entre el 90 y el
40 a. C. En el perfil 3 se estudió una unidad próxima al nivel II, arriba descrito, que se denominó IIIb, ya que ocupa una posición intermedia entre los niveles basales del siguiente perfil. En el perfil 4, de unos 50 $\mathrm{cm}$ de potencia, se estudió una secuencia de tres niveles de relleno superpuestos (IIIa, IIIc y IIId).

El nivel Illa ocupa los 5 primeros centímetros del perfil 4 por encima del nivel anterior. Está constituido por arcillas con escasas arenas y limos de color gris claro (10YR 7/2). El porcentaje de arcillas es elevado, por encima del $40 \%$. Ello indica un predominio absoluto de los procesos de decantación en aguas tranquilas, aunque se observan determinados picos en arenas finas y limos que vienen a alterar sus rasgos. La curva acumulativa y los parámetros texturales son idénticos a los del nivel basal. El porcentaje de materia orgánica se sitúa en torno al $0,43 \%$ y el de carbonatos en el $46,85 \%$.

El nivel IIlb es la base del relleno de la cubeta en el perfil 3, pero con una posición estratigráfica más elevada que la del perfil 4. Está formado por arcillas y limos con muy escasas arenas de color marrón amarillento claro (10YR 6/4). La distribución de los porcentajes por tamaños del sedimento permite observar una muy baja clasificación en las fracciones más finas. La curva acumulativa, la más tendida e hiperbólica de las estudiadas, indica que la decantación es de nuevo su rasgos más característico. Su color nos indica que predominan las condiciones de oxigenación, frente al anterior relleno, en el que fueron más importantes las de reducción. La materia orgánica 
Ferrer García, C., 2018. Dinámica fluvial durante el inicio del Holoceno superior en el... Boletín Geológico y Minero, 129 (1/2): $305-330$

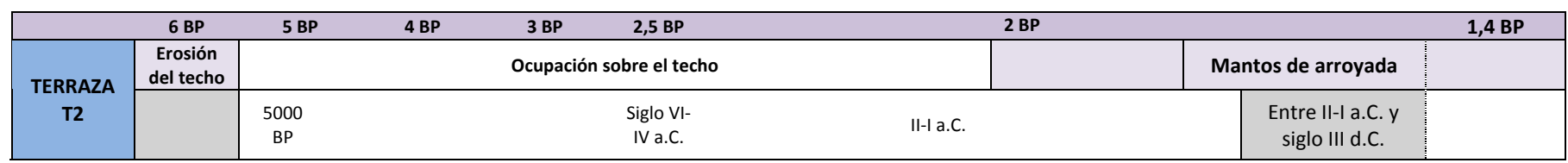

\begin{tabular}{|c|c|c|c|c|c|c|c|}
\hline $\begin{array}{c}\text { TERRAZA } \\
\text { T3 }\end{array}$ & & Unidad A & & Unidad B & & Unidad C & Unidad D \\
\cline { 2 - 6 } & & $\pm 4000 \mathrm{BP}$ & ¿? & s. V-IV a.C. & i? & i? & $\begin{array}{c}\text { s. II } \\
\text { d.C. }\end{array}$ \\
\hline
\end{tabular}

\begin{tabular}{|c|c|c|c|c|c|c|c|c|c|}
\hline \multirow{2}{*}{ EDAR } & & & & Nivel I & Nivel II y III & \multirow{2}{*}{$\begin{array}{c}\text { Nivel IVa y b } \\
20 \text { mitad s. I } \\
\text { d.C. }\end{array}$} & & \multirow{2}{*}{$\frac{\text { Nivel V }}{\text { s. II a V d.C. }}$} & \\
\hline & & & & III aC. & IIII a.C & & & & \\
\hline CLIMA & $\begin{array}{c}\text { Evento } 6 \\
\text { ka BP }\end{array}$ & & & & & & & Evento & \\
\hline
\end{tabular}

Tabla 2. Esquema resumen de la propuesta interpretativa de la secuencia cronoestratigráfica.

Table 2. Scheme of interpretation of sequence.

está presente en un porcentaje del 0,29\%, bajo para lo observado en sedimentos en los que predomina la decantación y el contexto arqueológico. El porcentaje de carbonato cálcico sí que es coherente con esta serie de sedimentos.

El nivel Illc se muestreó a cota $20-25 \mathrm{~cm}$ desde la base del perfil 4. Es de limos y arcillas de color gris marrón claro (10YR 6/2). La distribución textural revela un carácter bimodal, al producirse una muy relevante acumulación en torno a fracción arcillas y una secundaria en las arenas de tamaño 2-3 phi, lo que sería indicativo de la existencia de un transporte con energía variable que permite el desarrollo de dos modas en la fracción arenosa. La curva acumulativa sigmoidal y la presencia de tres claros segmentos en la curva semiprobabilística (reptación, saltación y suspensión) redundan en esta interpretación. Su formación respondería a arroyadas y fases de decantación que asociamos a depósitos de arroyadas difusas. El porcentaje de materia orgánica es idéntico al del subnivel basal, pero desciende ligeramente el de carbonato cálcico.

El nivel Illd se muestreó a cota $35-40 \mathrm{~cm}$ sobre la base, cerca del techo del perfil. Se trata de arenas y limos masivos formando agregados de color gris claro (10YR 7/2), con carbones de gran tamaño. Las gravas, muy escasas $(2,21 \%)$, de caliza subredondeada, se asocian a los elementos estructurales que amortizaban el conjunto.

La fracción arenosa es similar a la que conforman el resto de niveles III, aunque destaca en este caso la abundancia de microcarbones, que suponen toda la fracción arenosa por encima de 1,5 phi (más de 0,38 $\mathrm{mm}$ ). A pesar de ello, el porcentaje de materia orgá- nica es el mismo, $0,43 \%$. Los rasgos y parámetros texturales muestran una distribución que revela procesos sedimentarios similares a los que formaron el subnivel anterior, pero de menor energía y sin fracción relevante en reptación. Se trata de una escorrentía difusa de muy baja energía, con condiciones de nuevo de cierta hidromorfía.

El resto de niveles estudiados se superponen a la estructura arqueológica en el perfil 5 . Son posteriores a su obliteración en torno al 50-40 a. C. Se trata de dos pasadas de arenas que identificamos con el nivel IV y un estrato de limos y arcillas que sella el conjunto, el nivel $\mathrm{V}$.

El nivel IV está formado por dos subniveles muy parecidos desde el punto de vista estratigráfico, pero de diferente datación. El nivel IVa es un paquete de 10 a $12 \mathrm{~cm}$ de arenas de color marrón muy pálido (10YR $8 / 2$ ), que poseen una estratificación preferentemente horizontal, aunque se identifican en el perfil puntuales evidencias de estratificación cruzada. Coincide con la U.E. 103, que se apoya contra los muros del recinto por su paramento exterior y ofrece un conjunto material bastante numeroso y variado, incluyendo numerosos restos óseos, que lo datan en un momento avanzado del s. I d. C. El nivel IVb, coincidente con la U.E. 102, cubre la unidad anterior con contacto neto. Su sedimentación parece haberse producido al menos una centuria después del abandono del lugar, en el tránsito de los siglos I al II d. C.

Las muestras analizadas de estos subniveles presentan rasgos muy similares a los descritos en el nivel I. Se trata de nuevo de arenas rodadas y mates de cuarzo $(60 \%)$ y cuarzos angulosos brillantes $(35 \%)$, con algunas calizas rodadas. El porcentaje de materia 


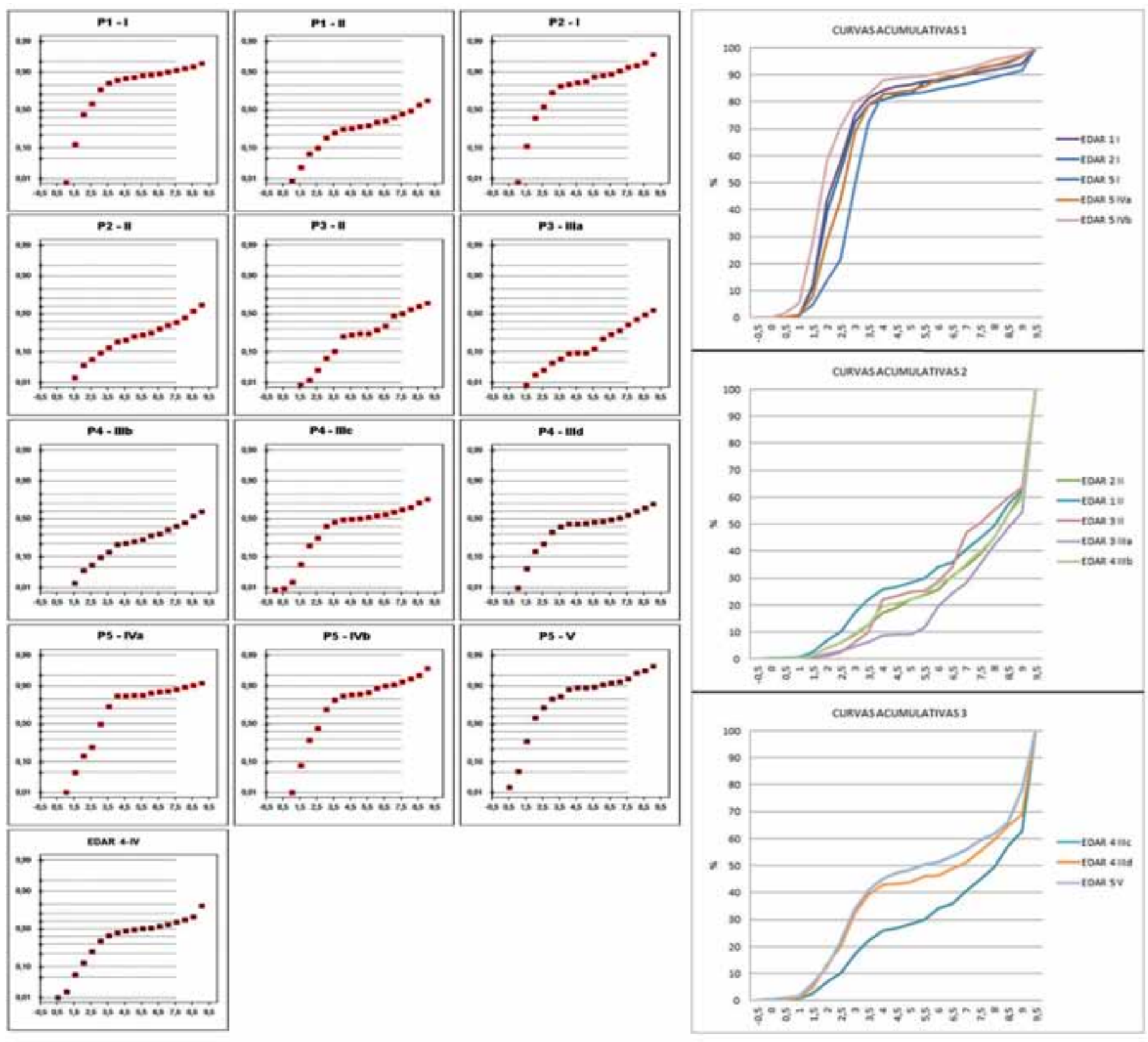

Figura 8. Curvas semiprobabilísticas (Visher, 1969) y curvas acumulativas del estudio sedimentológico. Escala de abscisas en unidades phi.

Figure 8. Log-probability curves (Visher, 1969) and cumulative curves of the sedimentological study. Abscissa scale in phi units.

orgánica y carbonato cálcico son de los más bajos de la serie (suponen menos del $0,1 \%$ y en torno al $23 \%$ respectivamente). La distribución textural de estas arenas muestra dos picos en 2 y 3 phi. Sus parámetros emparentan con los del nivel I, aunque su clasificación es algo más elevada. La fracción fina, en torno al $10 \%$, es preferentemente limosa y no conforma una clara cola, lo que parece indicar que no ha habido procesos de transporte en suspensión relevantes en su formación. El menor tamaño medio observado podría acerca el sedimento a procesos eólicos, pero sus rasgos morfoscópicos y la curva semiprobabilística han sido interpretados de nuevo como fluviales.

El nivel $V$ es un depósito de fracciones finas, limoarcillas, de unos $55 \mathrm{~cm}$ de espesor, formado por un sedimento de textura franca, bastante compacto y homogéneo, de color amarillo marrón (10YR 6/6) con algunos manchones de tono más oscuro (10YR 5/4). Cubre las unidades arriba citadas e incluye abundante material arqueológico de varios periodos desde 
época ibérica plena. Una cisterna caminera alto imperial abierta en este y los anteriores niveles aporta una datación ante quem: Partiendo de que se trata de una estructura enterrada en uso, podemos proponer la deposición de hasta $47 \mathrm{~cm}$ de nivel antes de su construcción, entre el siglo II y el siglo V d. C. (Segura et al., 2012).

En su análisis textural se observa como la fracción principal es la de las arcillas, con una moda secundaria relevante en las arenas en torno a 3 phi. Es de muy baja clasificación, platocurtosis y asimetría moderadamente negativa, rasgos que la separan de los sedimentos arenosos y la acercan a las características de los sedimentos que constituyen el relleno final de la cubeta central. También las curvas probabilística y acumulativa, sigmoidal, son muy similares a las presentadas por estas muestras, y confirman la existencia de procesos deposicionales por exceso de carga en dos o más fases. Podría tratarse de un sedimento bimodal o polimodal asociado a flujos hídricos de energía variable, tipo glacis o llano de inundación. EI porcentaje de materia orgánica es moderadamente elevado, un $0,36 \%$. El de carbonatos es el más alto del conjunto de muestras estudiadas.

\section{Discusión}

El conjunto de datos procedente del estudio de las terrazas fluviales de las Agualejas y del yacimiento arqueológico de EDAR permite llevar a cabo una propuesta cronoestratigráfica para la formación de los depósitos sedimentarios de este sector de la cuenca del río Vinalopó (Fig. 9. Tabla 2). Las formaciones edáficas y arenosas identificadas en el techo de la terraza T2 parecen señalar hacia una dinámica ambiental muy contrastada al final del Holoceno inferior e inicios del Holoceno medio, aunque será necesario un análisis específico y detallado para obtener conclusiones relevantes al respecto. La superficie erosiva sobre la que se asientan los poblados y estructuras desde el 5000 al 2200 BP informa de procesos de desmantelamiento de su techo antes de la primera fecha.

Tras el encajamiento de esta terraza, posiblemente en el Holoceno medio, se habrían reiniciado los procesos de acreción y tendría lugar el inicio de la formación de la terraza T3. Las cuatro unidades superpuestas identificadas tendrían una datación coherente según el principio de sucesión estratigráfica. Gran parte de la primera unidad, 1, sería contemporánea o posterior a la Edad del Bronce, del $4000 \mathrm{BP}$ en adelante (Fig. 9). La formación de la segunda, la unidad 2, coincidiría en parte con el periodo lbérico pleno, el Ibero-romano y el Romano alto imperial. La tercera se construyó en la transición entre el Alto y el Bajo Imperio. Finalmente, la última unidad tendría su desarrollo desde época bajo imperial en adelante.

Los procesos de agradación que conforman las unidades 1 y 2 coincidirían, al menos en parte, con fases de intensa ocupación humana que se desarroIlaron en la Edad del Bronce y entre los siglos VI y III a. C. La construcción de una acequia en el siglo II d. C. parece indicar que los procesos sedimentarios se habrían ralentizado en una fase ambiental de relativa estabilidad.

Es en la parte alta de esta secuencia fluvial en la que se inserta la estratigrafía del yacimiento de EDAR, que viene a aportar una visión más detallada del periodo que va desde el s. III a. C. al II d. C. EI estrato basal está constituido por arenas bien clasificadas de un medio sedimentario fluvial de hasta el s. III a. C., que se interpretan como asociadas a una fase de agradación lenta. En contraste, el nivel subsiguiente responde a un modelo sedimentario totalmente distinto en el que se dan procesos de decantación en condiciones de hidromorfía estacional o continua y moderado porcentaje de materia orgánica, que hacen que los sedimentos presenten tonos de bajo croma asociada a procesos de reducción. La datación de este nivel permite establecer que el ambiente hidromorfo se habría formado al menos desde el siglo II a. C. (entre el 180 y el 150 a. C.) y habría que asociarlo con una fase de ralentización o cese de los procesos de acreción. Sondeos llevados a cabo en la zona de EDAR antes de la excavación muestran que el área donde aflora es relativamente extensa en el contexto de la terraza T3 (Segura et al., 2012).

La topografía de esta unidad sedimentaria está alterada en el yacimiento por la apertura de una pequeña cubeta antrópica. Se abrió entre el 100 y el 80 a. C. con el objeto de que aflorara el agua en el área delimitada por los muros de una estructura, lo que pudiera indicar un descenso de los niveles freáticos. Los rellenos basales de la cubeta documentados en contexto arqueológico se habrían formado con posterioridad a la construcción de la citada estructura. Los primeros indican que se producen de nuevo procesos de decantación e hidromorfía. Destaca el hecho de que en el caso del segundo relleno el color expresa unas condiciones de oxidación que pudieran ser evidencia de un cambio sutil, un nuevo y puntual descenso del nivel freático. Los rellenos superiores se formaron antes del 50-40 a. C. en condiciones sedimentarias diferentes, ya que son el resultado de escorrentías de muy baja energía. Estas pudieran estar en relación de nuevo con pequeños cambios en las condiciones ambientales. Los rasgos del primer subnivel 
Terraza 2

Terraza 3
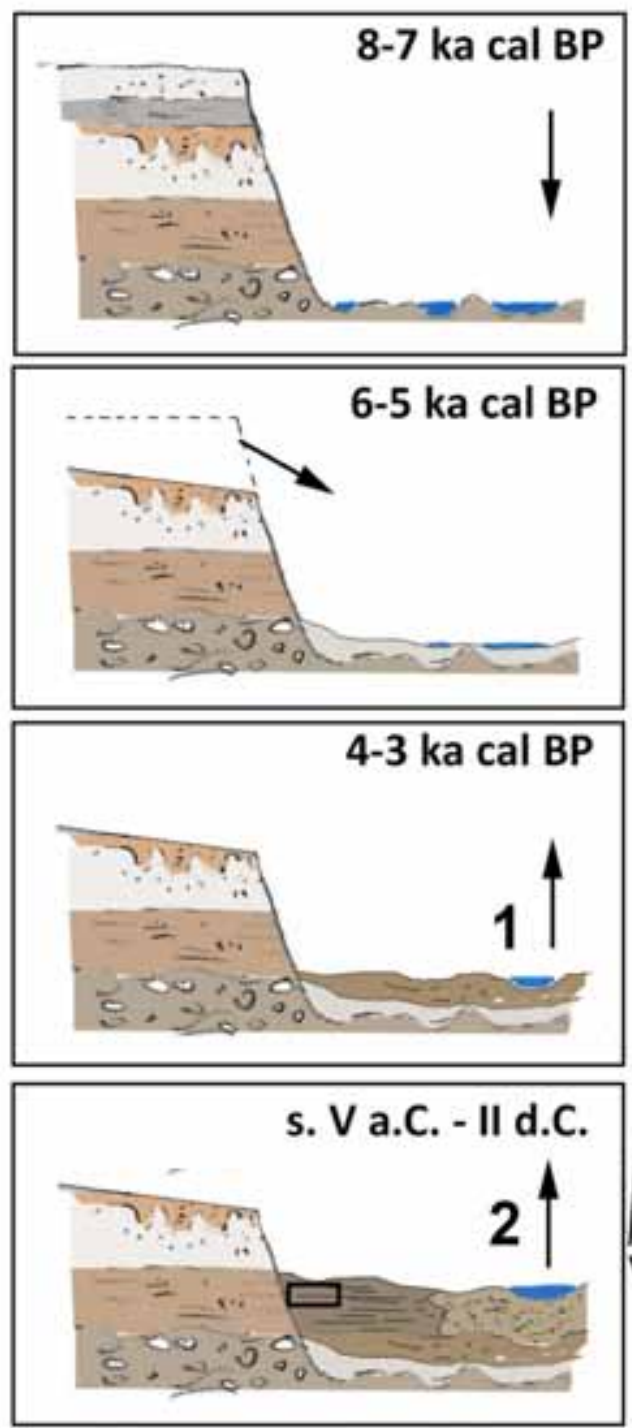

Terraza 2

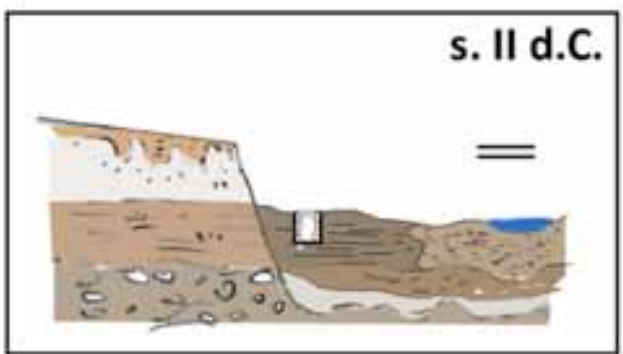

Unidad 2 Terraza 3
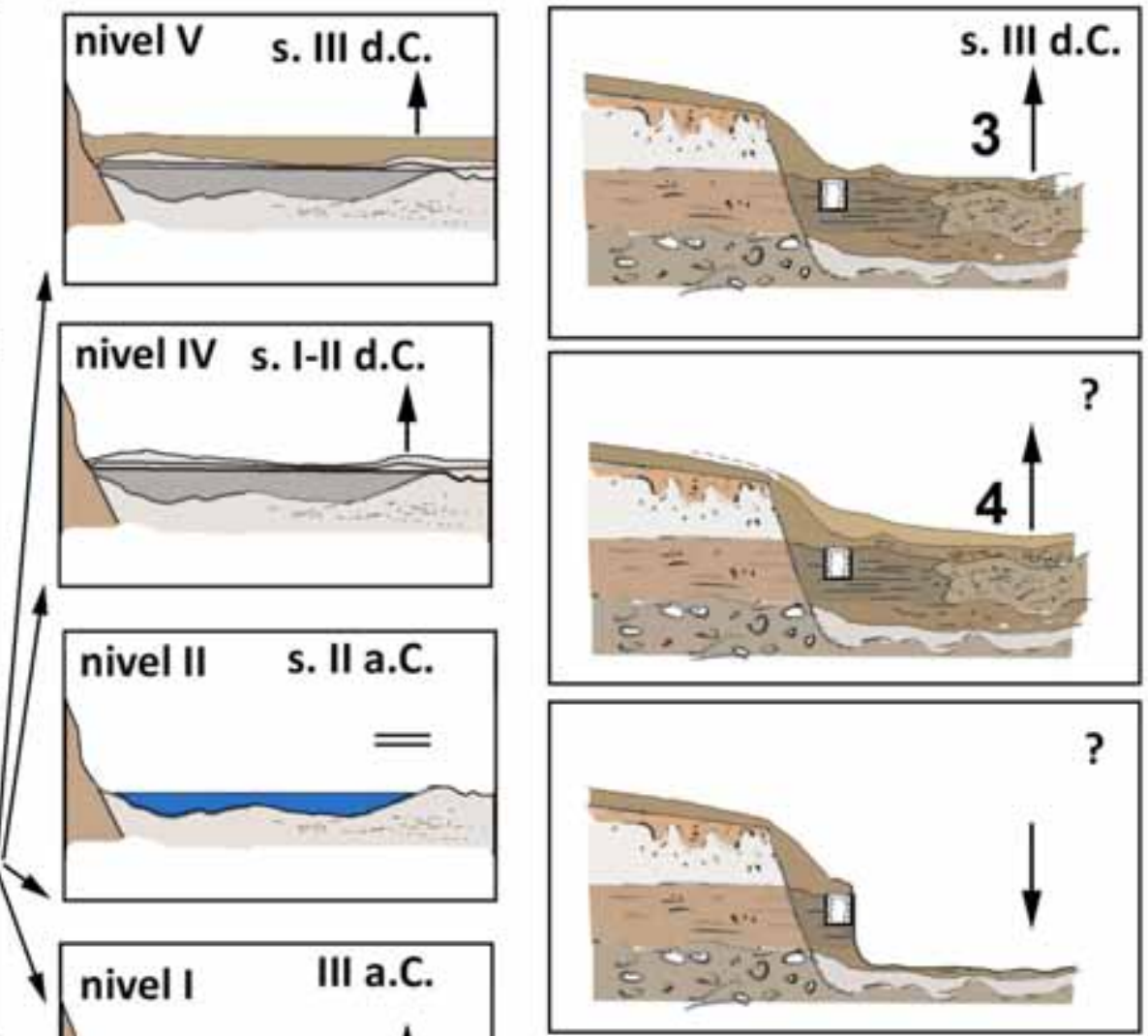

Figura 9. Esquema de la evolución del techo de la terraza T2 y de la formación de la terrazaT3. La flecha hacia arriba indica agradación, la flecha hacia abajo encajamiento, el símbolo de igual, indica estabilidad en el sistema sedimentario, la flecha oblicua indica erosión en el techo de la terraza T2. Los números del 1 al 4 junto a las fechas hacia arriba indica la construcción de las unidades sedimentarias descritas en la terraza T3.

Figure 9. Scheme of the T2 roof terrace evolution and scheme of the T3 terrace formation. The upper arrow indicates aggradation, the lower arrow encasement, equals symbol indicates stability in the sedimentary system, and the oblique arrow indicates the erosion at the top of the T2 terrace. The numbers from 1 to 4 indicate the construction of the sedimentary units described in the T3 terrace.

parecen indicar que se trata de un sedimento depositado en condiciones subaéreas; no así en el nivel que sella la secuencia, que vuelve a tener rasgos cromáticos anaeróbicos.

Las formaciones de arenas sobre el edificio amortizado se corresponden con un sedimento que empa- renta con el basal. El primer subnivel se depositó en un momento avanzado del siglo I d. C. y el segundo en el tránsito entre el siglo I y el II d. C. Son sedimentos fluviales que formarían parte del techo de la unidad 2 de la terraza T3. Sus características indicarían que se trata de procesos de agradación lenta. Con 
posterioridad se depositan sedimentos de llano de inundación o glacis entre los siglos II y el V d. C.

La fase de estabilidad descrita en EDAR se asociaría en parte con la documentada a techo de la unidad 2 de la terraza T3. En el contexto de esta terraza, la subsiguiente unidad 3 se inicia con un conjunto de sedimentos de colada, expresión de un evento de activa morfogénesis posterior a la primera mitad del siglo II d. C. Por su parte, los mantos de arroyada sobre la terraza antigua T2, que ponemos en relación con los sedimentos que forman el techo de EDAR, se habrían formado entre el siglo I a. C. y la primera mitad del siglo III d. C. en la villa de las Agualejas. Estos procesos podrían ser coincidentes en el tiempo y expresión de un evento sedimentario singular que fue capaz de construir, entre la segunda mitad del siglo II y del III d. C., además de esta unidad de la terraza T3, el depósito sedimentario sobre la terraza T2, tras 3000 años sin apenas actividad sedimentaria. La unidad 4 sería algo posterior, se habría formado en época plenamente bajo imperial / altomedieval, y pudo individualizarse a partir del siglo $\mathrm{V}$ y $\mathrm{VI} \mathrm{d}$. C.

\section{Interpretación: factores climáticos y presión humana}

La erosión documentada a techo de la terraza T2, anterior al $5000 \mathrm{BP}$, tiene una difícil interpretación paleoambiental. Procesos similares en la cuenca del río Guadalete se interpretaron en la llanura de inundación como resultado de un muy intenso desplazamiento del sistema de meandros, es decir, resultado de causas puramente dinámicas (Wolf et al., 2014). También pudiera ser el resultado de procesos asociados a una activa morfogénesis, que en medios fluviales semiáridos se asociarían a fases ambientales áridas, con frecuencia en forma de cambios climáticos rápidos (Faust et al, 2004, Zielhofer et al., 2004). Los modelos vigentes proponen el declive del bosque en el Mediterráneo occidental a partir del 6400/6300 BP (Frigola et al., 2007, Fletcher and Sánchez Goñi, 2008), aunque son pocas las evidencias que señalan hacia condiciones de marcada aridez antes del 5500 BP (Fernández et al., 2007). El clima del Este peninsular sería de rasgos plenamente mediterráneos desde el 5,0 ka cal BP (Dorado Valiño et al., 2002, Carrión et al., 2003, Pantaleón-Cano et al., 2003, Aranbarri et al., 2014, Oliva et al., 2009, García-Alix et al, 2012). Es posible pues pensar en un evento árido singular como responsable de este proceso, por ejemplo el documentado en torno al $6000 \mathrm{BP}$ a escala global y regional (Bond et al., 2001, Frigola et al., 2007, Fletcher and Zielhofer, 2013).

El esquema propuesto para la terraza T3 coincide, aunque de manera genérica, con la dinámica de los sistemas fluviales de medios semiáridos peninsulares hasta ahora estudiados. La formación de la unidad basal debió producirse en un contexto ambiental árido y con una intensa ocupación de la cuenca por parte de las sociedades de la Edad del Bronce. La unidad 2 en cambio, se desarrolló en su mayor parte en el contexto de una fase climáticamente fresca $y$ húmeda que se entendería desde $\pm 2,6$ ka BP hasta más allá del cambio de Era (Martín-Puertas et al., 2008, Oliva y Gómez-Ortiz, 2012, Currás et al., 2012, Gutiérrez et al., 2013, Jiménez-Moreno et al., 2013, Corella et al., 2013, e.g.), también documentada en medios fluviales y aluviales con la formación de suelos y la estabilidad ambiental (Wolf et al., 2014). Aunque se han descrito procesos de aluvionamiento que pudieran estar en relación con la presión antrópica sobre los sistemas geomorfológicos desde el inicio de esta fase (Constante et al., 2010, Bellin et al, 2013), la mayor parte de los trabajos proponen que la influencia de las actividades humanas en los sistemas fluviales se habría producido ya en época histórica, a partir del cambio de Era (Calmel-Avila, 2000, Faust et al., 2000, 2004 y 2015, Carrión et al., 2003, Schulte, 2002, Benito et al., 2008, Sancho et al., 2008, Schulte et al., 2008, Uribellarea and Benito, 2008, Zielhofer and Faust, 2008, García-Ruiz, 2010, Constante et al., 2010, Currás et al., 2012, Wolf et al., 2013).

En nuestro caso existe una correlación muy interesante entre las fases de intensa ocupación humana y la acreción fluvial en épocas Ibérica plena y Romana alto imperial, con la formación de barras y fondos de canal de arena desde al menos el siglo V hasta el III a. C. y durante el siglo I d. C. También entre el descenso de la presión antrópica entre los siglos III y I a. C. y el freno de estos procesos deposicionales. Estas relaciones pueden ser interpretadas como evidencia de un impacto directo sobre el sistema fluvial de la presión humana, aunque sólo en el caso de las formaciones sedimentarias de EDAR, y a partir del siglo III a. C., tendríamos una datación suficientemente contrastada para los procesos. Así, el abandono de las explotaciones agrarias en un contexto ambiental benigno permitió la recuperación de la cubierta vegetal y el cese de los procesos de acreción a partir del siglo II a. C., y los cambios acaecidos con la romanización plena, en el cambio de Era, produjeron importantes transformaciones en el paisaje que dieron lugar a la reactivación de los procesos de acreción en el siglo I d. C.

Esta correlación no sería tan clara para el siglo Il d. C., periodo en el que, con la perduración de las condiciones ambientales favorables y el moderadamente intenso uso del territorio, se observa cierta estabili- 
dad en la dinámica fluvial. La ralentización de los procesos sedimentarios pudiera responder a la recuperación o reequilibrio del sistema tras el primer impacto de las roturaciones romanas alto imperiales. Somos conscientes de la falta de datos consistentes para mantener esta afirmación, que conllevaría que el sistema sedimentario posee cierta capacidad para absorber las perturbaciones, pudiendo regresar a su estado original una vez superado su primer impacto, fenómeno ya descrito para sistemas dinámicos fluviales como resiliencia (Church et al., 2012).

La formación de la unidad 3 de la terraza T3, las arroyadas difusas del techo de EDAR y el nivel de aluvión sobre la terraza T2 marcan el final de la fase de estabilidad sedimentaria propuesta. Creemos que estos depósitos son expresión de un evento de activa morfogénesis datado en una época alto imperial más avanzada, entre la segunda mitad del siglo II y la primera del siglo III d. C. En este caso tampoco existe una relación directa entre procesos sedimentarios y acción humana, ya que las evidencias arqueológicas de una intensificación de la presión sobre el medio presentan cierto desfase en su datación. Efectivamente, en la ocupación de este territorio se habría producido un cambio significativo a partir de la segunda mitad del siglo III, y en particular durante el siglo IV d. C. (Tendero Porras et al., 2014, Molina Mas, 2015). En cambio, se ha documentado un evento climático árido a partir del 1,8 ka cal BP que pone fin a la fase húmeda, con el que pudiera estar relacionado. Este se ha descrito tanto en los modelos globales (Wanner et al., 2011), como en los peninsulares (Cacho et al., 2001, Frigola et al., 2007, Valero-Garcés et al., 2008, Calmel-Avila et al., 2009, Bellin et al., 2013, Fletcher et al., 2013, Jiménez-Moreno et al., 2013). Se trataría pues de un proceso vinculado no a la acción humana sino a un cambio climático rápido.

Por otro lado, cabe destacar que en periodos breves se han observado cambios sutiles, por ejemplo dentro de la fase de formación de sedimentos decantados en EDAR, en forma de descenso de la hidromorfía o de los procesos de decantación durante el siglo I a. C., que podrían indicar una muy elevada irregularidad climática.

\section{Conclusiones}

A la luz de lo expuesto es posible afirmar que el llano de Monforte ha tenido una dinámica sedimentaria muy activa a lo largo de los últimos milenios motivada por causas climáticas a partir del 6000/5000 cal BP, y en el marco de la interacción compleja de la acción humana a partir del 4000 cal BP. Se intuye un impac- to antrópico directo en el sistema sedimentario en la Edad del Bronce, coincidiendo con un periodo ambiental de marcada aridez, y en época Ibérica, condiciendo con rasgos climáticos que favorecen la biostasia que se extenderían más allá del siglo II d. C. Este impacto se hace más evidente en épocas Ibero-romana y Romana, cuando el descenso de la presión humana tuvo un efecto positivo en el sistema fluvial, ralentizando los procesos sedimentarios. A partir del cambio de Era las profundas transformaciones en el territorio afectaron al equilibrio del sistema sedimentario y produjeron una lenta acreción en el medio fluvial. La estabilización que el estudio intuye en torno al siglo II d. C., al final de la fase húmeda, aún bajo una continua presión humana, indicaría que el sistema sedimentario fluvial posee cierta resiliencia.

En el periodoTardorromano se observa un desfase entre procesos de agradación e incremento de la presión humana que impide establecer una relación directa entre ambas variables. Ello nos lleva a proponer que estos procesos sedimentarios pudieran responder a los cambios ambientales producidos tras el final de la fase húmeda o estar en relación con un evento árido. Con todo, cabe recordar que las características de los proxies secundarios que estudiamos y la falta de una cronología de alta resolución no nos permiten una aproximación más fina a la cuestión.

\section{Referencias}

Abad Casal, L. and Alberola Belda, E. 1990. Las Agualejas. Monforte del Cid, Vinalopó Mitjà. Memòries Arqueològiques de Salvament a la Comunitat Valenciana, 1984-1988, II. Intervencions rurals, Valencia, 74-76.

Abad Casal, L. and Sala Sellés, F. 1992. Las necrópolis ibéricas del área de Levante. Congreso de Arqueología Ibérica: las necrópolis, Madrid, 145-167.

Abad Casal, L., Sala, F. and Alberola, I. 1998. La necrópolis y el área sacra ibéricos de las Agualejas (Monforte del Cid, Alicante), Lucentum, 14-16, 7-18.

Alfaro, P., Andreu, J. M., Estévez, A., Tent-Manclús, J. E. and Yébenes A. 2004. Geología de Alicante. AEPECTUniversidad de Alicante, 267 pp.

Aranbarri, J., González-Sampériz, P., Valero-Garcés, B., Moreno, A., Gil-Romera, G., Sevilla-Callejo, M., García Prieto, E., Di Rita, F., Mata, M.P. Morellón, M., Magri, D., Rodríguez-Lázaro, J., Carrión and J. S. 2014. Rapid climatic changes and resilient vegetation during the Lateglacial and Holocene in a continental region of south-western Europe. Global and Planetary Change, $114,50-65$.

Badal, E., Martí Oliver, B. and Pérez Ripoll, M. 2012. From agricultural to pastoral use: changes in neolithic landscape at Cova de I'Or (Alicante, Spain). Saguntum, 13, 75-84. 
Bal, M.C., Pelachs, A., Perez-Obiol, R., Julia, R. and Cunill, R. 2011. Fire history and human activities during the last 3300cal yr BP in Spain's Central Pyrenees: The case of the l'Estany de Burg. Palaeogeography, Palaeoclimatology, Palaeoecology, 300(1), 179-190.

Bellin, N., Vanacker, V. and De Baets, S. 2013. Anthropogenic and climatic impact on Holocene sediment dynamics in SE Spain: a review. Quaternary International, 308, 112129.

Benito, G., Rico, M., Thorndycraft, V. R., Sánchez-Moya, Y., Sopeña, A., Díez-Herrero, A. and Jiménez, A. 2006. Palaeoflood records applied to assess dam safety in SE Spain. In: Ferreira, Alves, Leal and Cardoso (eds.). River Flow 2006. Taylor and Francis Group, 2113-2120. http://digital.csic.es/bitstream/10261/38547/1/benito_et_a I_2006_river_flow.pdf

Benito, G., Thorndycraft, V.R., Rico, M., Sánchez-Moya, Y. and Sopeña, A. 2008. Palaeoflood and floodplain records from Spain: evidence for long-term climate variability and environmental changes. Geomorphology, 101 (1), 68-77.

Benito, G., Macklin, M.G, Cohen, K.M. and Herget, J. 2013. Hydrological EXtreme Events in Changing Climate: The HEX Events project. Poster 8th IAG International Conference on Geomorphology - August 27th to 31st, 2013.

Blott, S. and Pye, K. 2001. Gradistat: grain size distribution and statistics package for the analysis of unconsolidated sediment. Earth, Surface Processes and Landforms, 26, 1237-1248.

Blott, S.J. and Pye, K. 2012. Particle size scales and classification of sediment types based on particle size distributions: Review and recommended procedures. Sedimentology, 59(7), 2071-2096.

Bond, G., Kromer, B., Beer, J., Muscheler, R., Evans, M.N., Showers. W., Hoffmann, S., Lotti-Bond, R., Hajdas, I. and Bonani, G. 2001. Persistent Solar Influence on North Atlantic Climate during the Holocene. Science, 294, 2130-2136.

Boggs, S. 2009. Petrology of sedimentary rocks. Cambridge University Press.

Burillo, F. and Peña, J.L 1984. Clima, geomorfología y ocupación humana. Introducción a un planteamiento metodológico. I Jornadas Metod. Inv. Prehist. Soria, 91-102.

Burillo, F., Gutiérrez, M. and Peña, J.L. 1985. Datación arqueológica de deformaciones tectónicas en vertientes holocenas de Sierra Palomera (Cordillera Ibérica centroriental). Actas da I Reunião do Quaternário Ibérico. Lisboa, 2, 355-366.

Butzer, K.W. 1982. Archaeology as human ecology: method and theory for a contextual approach. Cambridge University Press.

Butzer, K.W. 2005. Environmental history in the Mediterranean world: cross-disciplinary investigation of cause-and-effect for degradation and soil erosion. Journal of Archaeological Science, 32(12), 1773-1800.

Cacho, I., Grimalt, J.O., Canals, M., Sbaffi, L., Shackleton, N.J., Schönfeld, J. and Zahn, R. 2001. Variability of the western Mediterranean Sea surface temperature during the last 25,000 years and its connection with the
Northern Hemisphere climatic changes. Paleoceanography, 16 (1), 40-52.

Calmel-Avila, M. 2000. Procesos hídricos holocenos en el bajo Guadalentín (Murcia, SE España). Cuaternario y Geomorfología, 14, 65-78.

Calmel-Avila, M., Silva, P.G., Bardaji, T., Goy, J.L. and Zazo, C. 2009. Drainage system inversion in the Guadalentín Depression during the Late Pleistocene-Holocene (Murcia, Spain). In: Romero Díaz, M.A., Belmonte Serrato, F., Alonso Sarria, F. and López Bermúdez, F. (coord.), Congreso Internacional Sobre Desertificación, 461-464

Carmona, P. and Ruiz, J.M. 2011. Historical morphogenesis of the Turia River coastal flood plain in the Mediterranean littoral of Spain. Catena, 86, 139-149.

Carrión, J.S., Sánchez-Gómez, P., Mota, J.F., YII, R. and Chaín, C. 2003. Holocene vegetation dynamics, fire and grazing in the Sierra de Gádor, southern Spain. The Holocene, 13(6), 839-849.

Carrión, J.S., Fuentes, N., González-Sampériz, P., Sánchez Quirante, L., Finlayson, J.C., Fernández, S. and Andrade, A. 2007. Holocene environmental change in a montane region of southern Europe with a long history of human settlement. Quaternary Science Reviews, 26 (11), 14551475.

Carrión, J.S., Fernández, S., González-Sampériz, P., GilRomera, G., Badal, E., Carrión-Marco, Y., López-Merino, L., López-Sáez, J.A., Fierro, E. and Burjachs, F. 2010. Expected trends and surprises in the Lateglacial and Holocene vegetation history of the Iberian Peninsula and Balearic Islands. Review of Palaeobotany and Palynology, 162 (3), 458-475.

Constante, A., Peña-Monné, J.L. and Muñoz, A. 2010. Alluvial geoarchaeology of an ephemeral stream: implications for Holocene landscape change in the central part of the Ebro Depression, Northeast Spain. Geoarchaeology 25(4), 475-496.

Constante, A., Peña, J.L., Muñoz, A. and Picazo, J. 2011. Climate and anthropogenic factors affecting alluvial fan development during the late Holocene in the central Ebro Valley, northeast Spain. The Holocene, 21 (2), 275286.

Clavero, P. 1977. Los climas de la región Valenciana. Doctoral dissertation. Universitat de Barcelona.

Cordier, S., Bridgland, D., Vandenberghe, J. and Harmand, D. 2014. Fluvial archives from past to present-Introduction. Boreas, 43 (2), 377-383.

Corella, J.P., Stefanova, V., El Anjoumi, A., Rico, E., Giralt, S., Moreno, A., Plata-Montero, A. and Valero-Garcés, B.L. 2013. A 2500-year multi-proxy reconstruction of climate change and human activities in northern Spain:The Lake Arreo record. Palaeogeography, Palaeoclimatology, Palaeoecology, 386, 555-568.

Cuenca Paya, A. and Walker, M.J. 1995. Terrazas fluviales en la zona bética de la Comunidad Valenciana. El Cuaternario del País Valenciano, 105-114.

Currás, A., Zamora, L., Reed, J.M., García-Soto, E., Ferrer, S., Armengol, X, Mezquita-Jaomes, F., Marqués, M.A., Riera, S. and Julià, R. 2012. Climate change and human impact in central Spain during Roman times: High-reso- 
Ferrer García, C., 2018. Dinámica fluvial durante el inicio del Holoceno superior en el... Boletín Geológico y Minero, 129 (1/2): $305-330$

lution multi-proxy analysis of a tufa lake record (Somolinos, $1280 \mathrm{~m}$ asl). Catena, 89, 31-53.

Dorado Valiño, M., Valdeolmillos Rodrıguez, A., Blanca Ruiz Zapata, M., José Gil Garcıa, M., y de Bustamante Gutiérrez, I. 2002. Climatic changes since the Late-glacial/Holocene transition in La Mancha Plain (South-central Iberian Peninsula, Spain) and their incidence on Las Tablas de Daimiel marshlands. Quaternary International, 93, 73-84.

Dupré, M. 1995. Cambios ambientales en el territorio valenciano. La palinología. El Cuaternario del País Valenciano. AEQUA-GVQ, 205-216.

Ejarque, A., Miras, Y., Riera, S., Palet, J.M. and Orengo, H. A. 2010. Testing micro-regional variability in the Holocene shaping of high mountain cultural landscapes: a palaeoenvironmental case-study in the eastern Pyrenees. Journal of Archaeological Science, 37 (7), 1468-1479.

Faust, D., Diaz del Olmo, F. and Baena Escudero, R. 2000. Soils in the Holocene alluvial sediments of the Rio Fraja valley, Spain: in situ or soil-sediments? Catena, 41, 133142.

Faust, D., Zielhofer, C., Baena, R. and Díaz del Olmo, F. 2004. High resolution fluvial record of late Holocene geomorphic change in northern Tunisia: climatic or human impact? Quaternary Science Reviews, 23, 1757-1775.

Faust, D., Zielhofer, C. and Carrión García, J.S. 2015. Fluvial dynamics in Spain - Significance for palaeoenvironmental reconstructions and landscape evolution in the Western Mediterranean. Dissertation zur Erlangung des akademischen Grades Doctor rerum naturalium (Dr. rer. nat.) vorgelegt von Dipl.-Geogr. Daniel Wolf. $<$ <http://www.qucosa.de/fileadmin/data/qucosa/documents/17741/Wolf_10032015.pdf>>

Ferme, L. C. and Huerta, R.P. 2014. Landscape and forest exploitation at the ancient Neolithic site of La Draga (Banyoles, Spain). The Holocene, 24 (3), 266-273.

Fernández López de Pablo, J., Gómez Puche, M.G. and Martínez-Ortí, A. 2011. Systematic consumption of nonmarine gastropods at open-air Mesolithic sites in the Iberian Mediterranean region. Quaternary International, 244 (1), 45-53.

Fernández López de Pablo, J., Gómez Puche, M.M. and Esquembre Bebia, M.A. 2014. Casa Corona (Villena, Alicante, Spain). In Sala, R. (ed.), Pleistocene and Holocene hunter gatherers in Iberia and the Gibraltar Strait: the current archaeological record. Universidad de Burgos Fundación Atapuerca, 331-337.

Fernández, S., Carrión, J.S., Fuentes, N., González-Sampériz, P., Gil, G., García-Martínez, M.S. and Riquelme, J.A. 2007. Palynology of Carihuela Cave, southern Spain: completing the record. Geobios, 40, 75-90.

Ferrer García, C. and Blázquez Morilla, A.M. 1999. El abanico del Vinalopó a lo largo del Holoceno superior: una aproximación geoarqueológica, Cuadernos de geografía, 65-66, 347-358.

Flemming, B.W. 2007. The influence of grain-size analysis methods and sediment mixing on curve shapes and textural parameters: implications for sediment trend analysis. Sedimentary Geology, 202(3), 425-435.
Fletcher, W.J. and Sánchez Goñi, M.F. 2008. Orbital- and suborbital-scale climate impacts on vegetation of the western Mediterranean basin over the last 48,000 yr. Quaternary Research, 70, 451-464.

Fletcher, W.J. and Zielhofer, C. 2013. Fragility of Western Mediterranean landscapes during Holocene rapid climate changes. Catena, 103, 16-29.

Fletcher, W.J., Debret, M. and Sánchez Goñi, M.F. 2013. MidHolocene emergence of a low-frequency millennial oscillation in western Mediterranean climate: Implications for past dynamics of the North Atlantic atmospheric westerlies. The Holocene, 23(2), 153-166.

Folk, R.L. aned Ward, W.C. 1957. Brazos River bar: a study in the significance of grain size parameters. Journal of Sedimentary Petrology, 27, 3-26

Frigola, J., Moreno, A., Cacho, I., Canals, M. Sierro, J.F., Flores, J.A., Grimalt, J.O. Hodell D.A. and Curtis, J.H. 2007. Holocene climate variability in the western Mediterranean region from a deepwater sediment record. Paleoceanography, $22 . \quad$ PA2209, doi:10.1029/2006PA001307

Fumanal, M.P. 1986. Las cuevas habitadas en el cuaternario reciente. Sedimentología y clima en el País Valenciano. Diputación de Valencia, Trabajos Varios, 83, 207 pp.

Fumanal, M.P. and Carmona, P. 1995. Paleosuelos pleistocenos en algunos enclaves del País Valenciano. El Cuaternario del País Valenciano, 125-134.

García Atiénzar, G. 2006. Abrigos, valles y pastores. Análisis espacial del paisaje pastoril en las tierras centro-meridionales valencianas. In: Grau Mira, I (ed.), La aplicación de los SIG en la Arqueología del Paisaje, Universidad de Alicante, 149-170.

García-Alix, A., Jimenez-Espejo, F.J., Lozano, J.A., JiménezMoreno, G., Martinez-Ruiz, F., Garcia Sanjuan, L., Aranda Jiménez, G., García Alfonso, E., Ruiz-Puertas, G. and Anderson, R.S. 2013. Anthropogenic impact and lead pollution throughout the Holocene in Southern Iberia. Science of the Total Environment, 449, 451-460.

García-Ruiz, J.M., Moreno, A., González Sampériz, P.G., Valero Garcés, B.L. and Martí Bono, C.E. 2010. La cronología del último ciclo glaciar en las montañas del sur de Europa: una revisión. Cuaternario y Geomorfología, 24 (1), 35-46.

Gil Olcina, A. 2009. Clima e hipótesis de cambio climático en la región geográfica del sureste ibérico. Investigaciones Geográficas (Esp), 49, 5-22.

Grau Mira, I. and Moratalla Javega, J. 1997. El poblamiento de época ibérica en el Alto Vinalopó. I Congreso del Vinalopó. Agua i Territorio, 215-240.

Gregory, K.J., Benito, G., Dikau, R., Golosov, V., Johnstone, E.C., Jones, J.A.A., Macklin, M.G., Parsons, A.J., Passmore, D. G., Poesen, J., Soja, R., Starkel, L., Thorndycraft, V.R. and Walling, D. E. 2006. Past hydrological events and global change. Hydrological Processes, 20, 199-204.

Gutiérrez, F., Valero-Garcés, B., Desir, G., GonzálezSamperíz, P., Gutiérrez, A., Linares, R., Zarroca, M., Moreno, A., Guerrero, J., Roqué, C., Arnald, L.J. and Demuro, M. 2013. Late Holocene evolution of playa lakes in the central Ebro depression base on geophysical sur- 
veys and morpho-stratigraphic analysis of lacustrine terraces. Geomorphology, 196, 177-197.

Gutiérrez-Elorza, M. and Peña-Monné, J.L. 1998. Geomorphology and late Holocene climatic change in Northeastern Spain. Geomorphology, 23 (2), 205-217.

Hartmann, D. and Flemming, B. 2007. From particle size to sediment dynamics: An introduction. Sedimentary Geology, 202 (3), 333-336.

Hernández Pérez, M.S. 1983. La metalurgia prehistórica en el Valle Medio del río Vinalopó (Alicante). Lucentum, 2, 17-42.

Hernandez Pérez, M.S. and Alberola Belda, E. 1988. Ledua (Novelda, Alacant): un yacimiento de llanura en el neolítico valenciano. Archivo de Prehistoria Levantina, 18, 149-158.

Jalut, G., Amat, A.E., i Mora, S.R., Fontugne, M., Mook, R., Bonnet, L. and Gauquelin, T. 1997. Holocene climatic changes in the western Mediterranean: installation of the Mediterranean climate. Comptes Rendus de I'Académie des Sciences - Series IIA. Earth and Planetary Science, 325(5), 327-334.

Jalut, G., Dedoubat, J.J., Fontugne, M. and Otto, T. 2009. Holocene circum-Mediterranean vegetation changes: climate forcing and human impact. Quaternary international, 200(1), 4-18.

Jiménez-Moreno, G., García-Alix, A., Hernández-Corbalán, M.D., Anderson, R.S. and Delgado-Huertas, A. 2013. Vegetation, fire, climate and human disturbance history in the southwestern Mediterranean area during the late Holocene. Quaternary Research 79, 110-122.

Julià, R., Negendank, J.F.W., Seret, G., Brauer, A., Burjachs, F., Endres, Ch., Giralt, S., Lobo, A., Parés, J.M., Roca, J.R. and Wansard, G. 2000. Origin and evolution of desertification in the Mediterranean environment in Spain. In: Balabanis, P. Peter, D. Ghazi A. and Tsogas, M. (ed.), Mediterranean Desertification: Research results and policy implications, 2, 67-76.

Jover Mestre, F.J. and López Padilla, J.A. 2004. 2110-1200 BC. Aportaciones al proceso histórico en la cuenca del río Vinalopó. In: Hernández-Pérez, J. (coords.), La Edad del Bronce en las tierras valencianas y zonas limítrofes (Villena, 2002). Instituto Juan Gil-Albert. Alicante, 285302.

Macklin, M.G., Johnstone, E. and Lewin, J. 2005. Pervasive and long-term forcing of Holocene river instability and flooding in Great Britain by centennial-scale climate change. The Holocene, 15 (7), 937-943.

Macklin, M.G., Lewin, J. and Woodward, J.C. 2012. The fluvial record of climate change. Philosophical Transactions. Series A, Mathematical, Physical and Engineering Sciences, 370(1966), 2143-2172.

Marinova, E., Kirleis, W. and Bittmann, F. 2012. Human landscapes and climate change during the Holocene. Veget Hist Archaeobot. DOI 10.1007/s00334-012-0361-3

Márquez, J.C. 2006. El Valle de Elda en la época romana: el mundo rural. In: Poveda, A.M. (coord.), Historia de Elda. I. De las cabañas a la villa (desde la Prehistoria hasta el siglo XVIII), Alicante, 75-96.

Martín-Puertas, C., Valero-Garcés, B.L., Mata, M. P., González-Sampériz, P., Bao, R., Moreno, A. and
Stefanova, V. 2008. Arid and humid phases in southern Spain during the last 4000 years: the Zonar Lake record, Cordoba. The Holocene, 18 (6), 907-921.

Martín-Puertas, C., Valero, B.L., Brauer, A., Mata, M.P., Delgado-Huertas, A. and Dulski, P. 2009. The Iberian Roman Humid Period (2600 2100 cal yr BP) in the Zóñar Lake varve record (Andalucía, southern Spain). Quaternary Research, 71, 108-120.

Martín-Puertas, C., Jiménez-Espejo, F., Martínez-Ruiz, F., Nieto-Moreno, V. Rodrigo, M., Mata, M.P.; Valero-Garcés, B.L. 2010. Late Holocene climate variability in the southwestern Mediterranean region: an integrated marine and terrestrial geochemical approach. Clim. Past, 6, 807816

McLaren, P. and Bowles, D. 1985. The effects of sediment transport on grain-size distributions. Journal of Sedimentary Research, 55 (4), 457-470.

Molina Mas, F.A. 2015. Redescubriendo la Villa Romana de La Agualeja. (Monforte del Cid, Alicante), 20 años después. Arqueología y Museos, 06, 247-253.

Molina Mas, F.A. and Ortega Pérez J.R. 2010. Excavación arqueológica en el Camino del Río, Monforte del Cid (Alicante). Polígono 16, Parcela 604. Memoria en elaboración.

Molina Vidal, J. 2002. La irrupción de Hispania en los movimientos socioeconómicos del Mediterráneo Occidental durante las Guerras Civiles. Gerión. Revista de Historia Antigua, 20 (1), 281-306.

Moratalla, J. 2005. El territorio meridional de la Contestania. Jornadas de Arqueología La Contestania Ibérica, treinta años después. Alicante, 91-118

Mycielska-Dowgiałło, E. and Ludwikowska-K dzia, M. 2011. Alternative interpretations of grain-size data from Quaternary deposits. Geologos, 17 (4), 189-203.

Munsell Color C.O. 2000. Munsell soil color charts. Gretabacbeth.

Olcina Cantos, J., Rico, A. and Paños, V. 2007. Un medio físico de aridez y contrastes con un río-rambla que otorga personalidad geográfica. In: Hermosilla, J. (ed.), Los riegos tradicionales en el Vinalopó, CHJ, 21-30.

Oliva, M., Ortiz, A. G., Schulte, L. and Salvador, F. 2009. Procesos periglaciares actuales en Sierra Nevada. Distribución y morfometría de los lóbulos de solifluxión. Nimbus. Revista de climatología, meteorología y paisaje, 23, 133-148.

Oliva, M. and Gómez-Ortiz, A. 2012. Late-Holocene environmental dynamics and climate variability in a Mediterranean high mountain environment (Sierra Nevada, Spain) inferred from lake sediments and historical sources. The Holocene, 22 (8), 915-927.

Ortega Pérez, J.R., Boronat Soler, J.D. and Morán Macías, J.A. 2008. Excavación arqueológica en las obras del Colector de Recogida de Aguas Pluviales (PP.KK. 1.0271.157). Partida de Los Baños. Monforte del Cid (Alicante). Actuaciones Arqueológicas en la Provincia de Alicante 2008, edición en CD, Sección de Arqueología del llustre Colegio Oficial de Doctores y Licenciados en Filosofía y Letras y en Ciencias de Alicante.

Pantaléon-Cano, J., YII, E.I., Pérez-Obiol, R. and Roure, J.M. 2003. Palynological evidence for vegetational history in 
Ferrer García, C., 2018. Dinámica fluvial durante el inicio del Holoceno superior en el... Boletín Geológico y Minero, 129 (1/2): $305-330$

semi-arid areas of the western Mediterranean (Almeria, Spain). The Holocene, 13 (1), 109-119.

Peña-Monné, J.L., Julián, A., Chueca, J., Echeverría, M.T. and Ángeles, G. 2004. Etapas de Evolución Holocena en el Valle del Río Huerva: Geomorfología y Geoarqueología. In: J.L. Peña, L.A. Longares and M. Sánchez (eds.), Geografía Física de Aragón: aspectos generales $y$ temáticos. Universidad de ZaragozaInstitución Fernando el Católico, 289-302. <<http://age.ieg.csic.es/fisica/docs/025.pdf >>

Peña-Monné, J.L., Sancho, C. and Rubio, V. 2005. Aportaciones desde la Geomorfología y la Geoarqueología al Conocimiento de los Paleoambientes Holocenos del NE de España. Actas $X$ Coloquio Ibérico de Geografía. Évora, Portugal. <<http://www.apgeo.pt/ files/docs/CD_X_Coloquio_Iberico_Geografia/pdfs/077.p $\mathrm{df}>>$

Pérez Cueva, A. J. 1994. Atlas climàtic de la Comunitat Valenciana (1961-1990). Generalitat Valenciana, Valencia, $205 \mathrm{pp}$.

Pérez Obiol, R., Jalut, G., Juliá, R., Pèlachs Mañosa, A., Iriarte Chiapusso, M.J., Otto, T. and Hernández Beloqui, B. 2011. Mid-Holocene vegetation and climatic history of the Iberian Peninsula. The Holocene, 21 (1), 75-93.

Pye, K. 1987. Aeolian Dust and Dust Deposits. Academic Press, London, $334 \mathrm{pp}$.

Ramos Muñoz, J. and Pérez Rodríguez, M. 2008. La transformación del medio natural en el entorno de la bahía y banda atlántica de Cádiz por sociedades cazadoras recolectoras, tribales comunitarias y clasistas iniciales. In: Arteaga, O. and Schulz, H.D. (eds.), La transformación del medio natural en el entorno de la Bahía de Cádiz. Revista Atlántica-Mediterránea de Prehistoria $y$ Arqueología Social, 10, 155-213.

Reineck, S. and Singh, I.B. 1980. Depositional sedimentary environments. Springer-Verlag, Berlin, 549 pp.

Revelles, J., Cho, S., Iriarte, E., Burjachs, F., Van Geel, B., Palomo, A. and Terradas, X. 2015. Mid-Holocene vegetation history and Neolithic land-use in the Lake Banyoles area (Girona, Spain). Palaeogeography, Palaeoclimatology, Palaeoecology, 435, 70-85.

Roberts, N., Brayshaw, D., Kuzucuo lu, C., Perez, R. and Sadori, L. 2011. The mid-holocene climatic transtion in the Mediterranean: causes and consequences. The Holocene, 21, 3-13.

Rodríguez-Estrella, T. 1977. Los grandes accidentes tectónicos de la provincia de Alicante. Boletín del Instituto Geológico y Minero, 88 (3 y 4), 183-214 y 273-299.

Rosselló i Verger, V.M. and Boira i Maiques, J.V. 1995. Geografia del Pais Valencià. Institució Valenciana d’Estudis i Investigació. Generalitat Valenciana, Valencia, 640 pp.

Rubiales, J. M., Morales-Molino, C., Álvarez, S.G. and García-Antón, M. 2012. Negative responses of highland pines to anthropogenic activities in inland Spain: a palaeoecological perspective. Vegetation history and archaeobotany, 21 (4-5), 397-412.

Sala Sellés, F., Abad Casal, L. and Alberola, E. 1999. La necrópolis y el área sacra ibéricos de "Las Agualejas" (Monforte del Cid, Alicante). Lucentum, XIV-XVI, 7-18.
Sancho, C., Peña, J.L., Muñoz, A., Benito, G., McDonald, E., Rhodes, E. J. and Longares, L. A. 2008. Holocene alluvial morphopedosedimentary record and environmental changes in the Bardenas Reales Natural Park (NE Spain). Catena, 73 (3), 225-238.

Schulte, L. 2002. Climatic and human influence on river systems and glacier fluctuations in southeast Spain since the Last Glacial Maximum. Quaternary International, 93, 85-100.

Schulte, L., Julià, R., Burjachs, F. and Hilgers, A. 2008. Middle Pleistocene to Holocene geochronology of the River Aguas terrace sequence (Iberian Peninsula): fluvial response to Mediterranean environmental change. Geomorphology, 98 (1), 13-33.

Segura, G, Moratalla, J., Aguado, F., Flor, J.M. and Tordera, F.F. 2012. Excavación arqueológico en la Parcela 604, polígono 16 (Monforte del Cid, Alicante). Memoria Final. Arquealia, 319 pp.

Shackley, M.I. (1975). Archaeological sediments: a survey of analytical methods. London and Boston, Butterwork, $168 \mathrm{pp}$.

Soil Survey Staff 2014. Soil Survey Field and Laboratory Methods Manual. Soil Survey Investigations Report No. 51, Version 2.0. In: Burt R. and Soil Survey Staff (ed.). U.S. Department of Agriculture, Natural Resources Conservation Service. http://www.nrcs.usda.gov /Internet/FSE_DOCUMENTS/stelprdb1244466.pdf

Stokes, M., Griffiths, J.S. and Mather, A. 2012. Palaeoflood estimates of Pleistocene coarse grained river terrace landforms (Río Almanzora, SE Spain). Geomorphology, 149, 11-26.

Tendero Porras, M., Ronda Femenia, A.M., Ramos Fernández, R., Ramos Molina, A., Peña Domínguez, D., and Abad Casal, L. 2014. Ciudades Romanas Valencianas. Ilici. In: Olcina Domènech, M. (ed.), Ciudades Romanas Valencianas. Actas de las Jornadas sobre Ciudades Romanas Valencianas. Actualidad de la investigación histórico arqueológica. Marq, 226-243.

Thorndycraft, V.R. and Benito G. 2006. Late Holocene fluvial chronology of Spain: The role of climatic variability and human impact. Catena, 66 (1-2), 34-41.

Tricart, J. 1965. Principes et Methodes de la Geomorphologie. Soil Science, 100(4), 300 pp.

Uribelarrea, D. and Benito, G. 2008. Fluvial changes of the Guadalquivir river during the Holocene in Córdoba (Southern Spain). Geomorphology, 100(1), 14-31.

Valero-Garcés, B.L. and Moreno, A. 2011. Iberian lacustrine sediment records: responses to past and recent global changes in the Mediterránean region. Journal of Paleolimnology, 46, 319-325.

Van Andel, T.H., Runnels, C.N. and Pope, K.O. 1986. Five Thousands Years of Land Use and Abuse in the Southern Argolid, Greece. Hesperia, 5, 103-128.

Van Zuidam, R.A. 1975. Geomorphology and Archaeology. Evidences of interrelation at historical sites in the Zaragoza region, Spain. Zeitschrift für Geomorphologie, 19 (3), 319-328.

Visher, G.S. 1969. Grain size distribution and depositional processes. J. Sed. Petrol, 39, 1074-1106.

Vita-Finzi, C. 1969. The Mediterranean valleys. Geological 
changes in historical times. Cambridge Univ. Press. London, $140 \mathrm{pp}$.

Walkley, A. and Black, I.A. 1934. An examination of the Degtjareff method for determining soil organic matter, and a proposed modification of the chromic acid titration method. Soil science, 37 (1), 29-38.

Wolf, D., Seim, A., Diaz del Olmo, F. and Faust, D. 2013. Late Quaternary fluvial dynamics of the Jarama River in central Spain. Quaternary International, 302, 17, 20-41.

Wolf, D. and Faust, D. 2014. Fluvial system response to external forcing and human impact - Late Pleistocene and Holocene fluvial dynamics of the lower Guadalete River in western Andalucía (Spain). Boreas, 43 (2), 422449.

Zapata, L., López-Sáez, J.A., Ruiz-Alonso, M., Linstädter, J., Pérez-Jordà, G., Morales, J., Kehl, M. and PeñaChocarro, L. 2013. Holocene environmental change and human impact in NE Morocco: Palaeobotanical evidence from Ifri Oudadane. The Holocene, 23(9), 1286-1296.

Zielhofer, C. and Faust, D. 2008. Mid and Late Holocene fluvial chronology of Tunisia. Quaternary Science Reviews, 27, 580-588.

Recibido: junio 2015

Revisado: febrero 2016

Aceptado: marzo 2016

Publicado: marzo 2018 